\title{
Modeling CO emission from hydrodynamic simulations of nearby spirals, starbursting mergers, and high-redshift galaxies
}

\author{
F. Bournaud ${ }^{1}$, E. Daddi ${ }^{1}$, A. Wei $\aleph^{2}$, F. Renaud ${ }^{1,3}$, C. Mastropietro ${ }^{1}$, and R. Teyssier ${ }^{4}$ \\ 1 Laboratoire AIM Paris-Saclay, CEA/IRFU/SAp, CNRS/INSU, Université Paris Diderot, 91191 Gif-sur-Yvette Cedex, France \\ e-mail: Frederic.Bournaud@cea.fr \\ 2 Max-Planck-Institut für Radioastronomie (MPIfR), Auf dem Hügel 16, 53121 Bonn, Germany \\ 3 Department of Physics, University of Surrey, Guildford GU2 7XH, UK \\ ${ }^{4}$ Institute for Computational Science, University of Zurich, 8057 Zürich, Switzerland
}

Received 29 September 2014 / Accepted 16 December 2014

ABSTRACT

\begin{abstract}
We model the intensity of emission lines from the $\mathrm{CO}$ molecule, based on hydrodynamic simulations of spirals, mergers, and highredshift galaxies with very high resolutions $\left(3 \mathrm{pc}\right.$ and $10^{3} M_{\odot}$ ) and detailed models for the phase-space structure of the interstellar gas including shock heating, stellar feedback processes, and galactic winds. The simulations are analyzed with a large velocity gradient (LVG) model to compute the local emission in various molecular lines in each resolution element, radiation transfer, opacity effect, and the intensity emerging from galaxies to generate synthetic spectra for various transitions of the $\mathrm{CO}$ molecule. This model reproduces the known properties of $\mathrm{CO}$ spectra and $\mathrm{CO}-$ to- $\mathrm{H}_{2}$ conversion factors in nearby spirals and starbursting major mergers. The high excitation of $\mathrm{CO}$ lines in mergers is dominated by an excess of high-density gas, and the high turbulent velocities and compression that create this dense gas excess result in broad linewidths and low $\mathrm{CO}$ intensity-to- $\mathrm{H}_{2}$ mass ratios. When applied to high-redshift gas-rich disks galaxies, the same model predicts that their $\mathrm{CO}-$ to- $\mathrm{H}_{2}$ conversion factor is almost as high as in nearby spirals, and much higher than in starbursting mergers. High-redshift disk galaxies contain giant star-forming clumps that host a high-excitation component associated to gas warmed by the spatially concentrated stellar feedback sources, although $\mathrm{CO}(1-0)$ to $\mathrm{CO}(3-2)$ emission is dominated overall by low-excitation gas around the densest clumps. These results generally highlight a strong dependence of $\mathrm{CO}$ excitation and the $\mathrm{CO}-$ to- $\mathrm{H}_{2}$ conversion factor on galaxy type, even at similar star formation rates or densities. The underlying processes are driven by the interstellar medium structure and turbulence and its response to stellar feedback, which depend on global galaxy structure and in turn affect the $\mathrm{CO}$ emission properties.
\end{abstract}

Key words. galaxies: ISM - galaxies: star formation

\section{Introduction}

Understanding the molecular gas content of galaxies at various stages of their formation is an increasingly important issue in the field of galaxy evolution, especially with high-resolution campaigns using radio interferometers (e.g., Daddi et al. 2010b; Tacconi et al. 2010, 2013; Combes et al. 2011; Sargent et al. 2012; Walter et al. 2014; Schinnerer et al. 2013; Meidt et al. 2013). In parallel, the last generations of cosmological simulations start resolving substructures in the interstellar gas reservoirs of galaxies with gas densities up to that of star-forming molecular clouds (Ceverino et al. 2012; Hopkins et al. 2014). Interpreting observations of the molecular tracers of the interstellar medium (ISM), including the CO molecule and comparing with theoretical predictions on the bulk of the molecular mass (i.e., the $\mathrm{H}_{2}$ molecule) remains highly uncertain. The variations in the excitation of the $\mathrm{CO}$ emission and the $\mathrm{CO}-$ to- $\mathrm{H}_{2}$ conversion factor are indeed very large, and their interpretation largely relies on arbitrary calibrations. The conversion factors often used to estimate the $\mathrm{H}_{2}$ mass depend on various factors, such as the metallicity (Israel 1997; Genzel et al. 2012). They also appear to vary with galaxy type in the nearby Universe (Downes \& Solomon 1998; Solomon \& Vanden Bout 2005), which led to the observation that various types of galaxies may also form their stars with very different efficiencies (Daddi et al. 2010a; Genzel et al. 2010) - a result questioned for depending on arbitrary assumptions on $\alpha_{\mathrm{CO}}$ (see, e.g., Feldmann et al. 2011; Papadopoulos et al. 2014; and Sargent et al. 2012, for detailed discussions).
An increasing amount of theoretical work is performed with analytic and numerical models, in particular to implement the $\mathrm{H}_{2}$ molecule formation in high-resolution simulations and/or to model the emission of the CO molecule (e.g., Narayanan et al. 2011, 2012; Narayanan \& Hopkins 2013; Krumholz \& Gnedin 2011; Feldmann et al. 2012a,b; Papadopoulos et al. 2012; Narayanan \& Krumholz 2014). In this paper, we introduce a novel approach combining large velocity gradient (LVG) analysis with high-resolution adaptive mesh refinement (AMR) simulations of various types of galaxies. These simulations ideally describe the ISM phase-space structure on small scales (down to a few pc) and up to very high densities $\left(\geq 10^{6} \mathrm{~cm}^{-3}\right)$, and LVG post-processing determines the emergent intensity of $\mathrm{CO}$ emission lines from this ISM structure.

The underlying assumption of this technique is that opacity effects occur mostly on relatively small scales (a few parsecs), because the low volume-filling factor of the molecular ISM makes it unlikely for two clouds to be found on a given line of sight with co-incident Doppler shifts to allow for largescale re-absorption. On small scales, depending on the (turbulent) kinematic structure of the clouds, the interstellar medium becomes transparent to its own emission owing to Doppler shifting of the molecular lines beyond a sufficient distance - generally a few pc or less, as determined from the kinematic structure of the AMR simulations coupled to the LVG model grids. These grids also determine the $\mathrm{CO}$ emissivity in various lines as a function of temperature, density, metallicity, and redshift (CMB temperature). 
We use this technique to analyze and compare simulations of various types of galaxies: nearby spiral galaxies with low gas fractions and secularly-evolving disks, high-redshift disk galaxies with high gas fractions and disk instabilities, and interaction/merging galaxies in a starburst (SB) phase, i.e. galaxies that have recently undergone a major increase in their star formation rate under the effect of a major interaction or merger. (Not all galaxy mergers undergo such phases, and these may last a shorter time than the merging process itself, Di Matteo et al. 2007; Ellison et al. 2013.) We probe the effect of galaxy type and structure on the $\mathrm{CO}$ excitation and $\alpha_{\mathrm{CO}}$ conversion factor, and to identify these effects we fix some other physical parameters. In particular, the metallicity is fixed at solar value (relatively massive galaxies at redshift $z=1-2$ are already significantly enriched anyways - Erb et al. 2006; Queyrel et al. 2012; Wuyts et al. 2014). We find that although they are very gas rich and unstable, high-redshift disks have global CO properties that are relatively similar to Milky Way-like spirals, however with locally substantial high-excitation components in their giant star-forming clumps. Even though they form stars about at the same rate as high-redshift disks, SB mergers have very different $\mathrm{CO}$ properties arising from a different smallscale ISM structure.

The simulations used in this work and the analysis technique are presented in Sect. 2. The results obtained for various types of galaxies are analyzed in Sect. 3, and they are compared to other theoretical and numerical models in Sect. 4. Throughout the manuscript, the $\mathrm{CO}$ luminosity-to- $\mathrm{H}_{2}$ mass conversion factor $\alpha_{\mathrm{CO}}$ is given in $M_{\odot}\left(\mathrm{K} \mathrm{km} \mathrm{s}^{-1} \mathrm{pc}^{2}\right)^{-1}$.

\section{Simulations and analysis}

\subsection{Simulation sample}

The three simulations used here were originally provided by different studies, but were performed with the same adaptive mesh refinement (AMR) code RAMSES (Teyssier 2002). They use similar spatial resolutions of 3.0 to $3.3 \mathrm{pc}$ and similar AMR refinement schemes and include the same physical ingredients: gas cooling at solar metallicity, heating with a ultraviolet background $^{1}$, and comprehensive modeling of stellar feedback processes from Renaud et al. (2013), including photo-ionization, radiation pressure, and Type-II supernovae. A complete description of the simulation methods can be found in Bournaud et al. (2014) or Renaud et al. (2014a).

We use three high-resolution simulations, which represent high-redshift disk galaxies, low-redshift spiral galaxies, and lowredshift starbursting mergers, all starting with similar baryonic masses of $6-7 \times 10^{10} M_{\odot}$ :

- a spiral representative of low-redshift disk galaxies with a gas fraction of $9 \%$ of the baryonic mass and a morphology dominated by spiral arms and small gas clouds forming mostly along the spiral arms. The star formation rate is 2-3 $M_{\odot} \mathrm{yr}^{-1}$. This is the control disk run from Renaud et al. (2014a,b).

- a high- $z$ disk model with high gas fraction (initially $60 \%$ of the baryonic mass, decreasing to $50-40 \%$ along the simulation through gas consumption), subject to violent disk instability and spontaneously taking an irregular morphology

\footnotetext{
1 We used a standard UV background that represents the Galaxy and low-redshift conditions. Redshift evolution of the UV background is neglected here, but likely to affect mostly the low-density phases of the ISM, rather than the molecular clouds that are mostly shielded from the UV background.
}

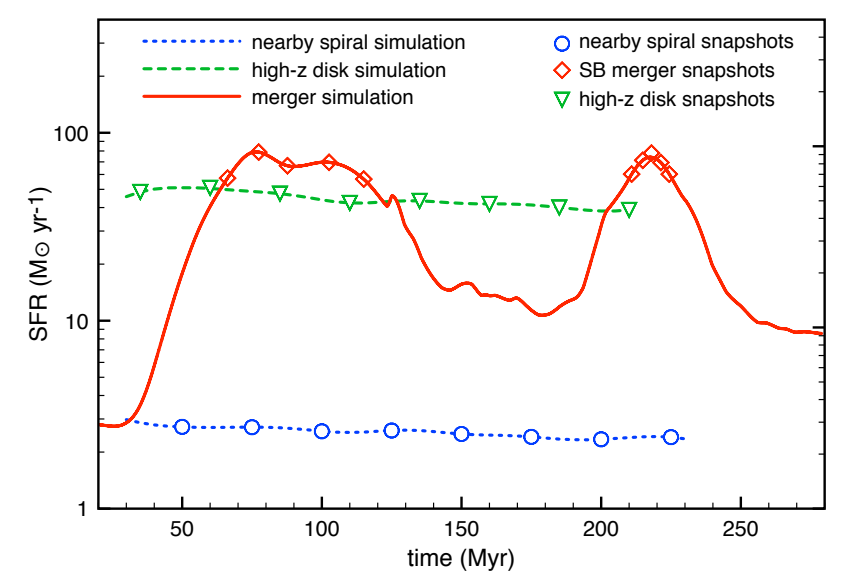

Fig. 1. Star formation rate as a function of time for the three galaxy models analyzed in this work. The snapshots/instants analyzed for each galaxy type are highlighted with various symbols. They are chosen randomly (about evenly spaced in time) for disks and only in starburst phases with elevated SFR for the major merger simulation. The corresponding sSFRs (averaged for each type) are given in Table 1.

Table 1. Physical properties of the three simulations representative of various galaxy types in this work.

\begin{tabular}{lcccc}
\hline \hline Model & $M_{*}$ & $f_{\text {gas }}$ & Redshift & $\langle s S F R\rangle$ \\
\hline spiral & $6.5 \times 10^{10} M_{\odot}$ & $9 \%$ & $z=0$ & $0.04 \mathrm{Gyr}^{-1}$ \\
SB merger & $6.5 \times 10^{10} M_{\odot}(\times 2)$ & $9 \%$ & $z=0$ & $0.63 \mathrm{Gyr}^{-1}$ \\
high- $z$ disk & $3.5 \times 10^{10} M_{\odot}$ & $60 \%$ & $z=2$ & $0.97 \mathrm{Gyr}^{-1}$ \\
\hline
\end{tabular}

Notes. The specific star formation rate (sSFR) indicated here is averaged over the snapshots analyzed for each galaxy type: random for the spiral and high- $z$ disk model, picked only in starbursting phases for the SB merger model. The stellar mass and gas fraction are the initial parameters of the models. A representative redshift is indicated for each model and is also used as an input parameter for the CMB temperature in the LVG grids.

dominated by young star-forming clumps, with high turbulent velocity dispersions and significant outflows, all consistent with observations: this is model G' 2 from Bournaud et al. (2014) and Perret et al. (2014), recomputed here at $1.7 \mathrm{pc}$ resolution for consistency with the low-redshift simulations. The star formation rate is consistent with the "main sequence" of star-forming galaxies at $z \approx 2$.

- a starbursting (SB) merger, produced by a major merger between two spiral galaxies similar to the spiral model used here. The chosen merger model, from Renaud et al. (2014a,b) has an orbit and a tidal field that is quite representative of mergers in $\Lambda$-CDM cosmology. The interaction triggers starburst events peaking at $80-100 M_{\odot} \mathrm{yr}^{-1}$ at the first pericentric encounter and during the final coalescence: the analyzed snapshots correspond to these phases when the merging system is actually starbursting about a strong LIRG or moderate ULIRG-like level, with an SFR ${ }^{2}$ in the $45-100 M_{\odot} \mathrm{yr}^{-1}$ range. Phases with more quiescent star formation are not considered as representative of an SB merger and ignored in the present analysis.

Table 1 summarizes the key properties of each simulation. The star formation rate evolution in each one is displayed in Fig. 1. We explore the properties of $\mathrm{CO}$ emission as a function of galaxy

\footnotetext{
2 Averaged over $10 \mathrm{Myr}$.
} 
type, rather than as a function of metallicity (see also the discussion in Sect. 4). We therefore assume solar metallicity for all cases, since massive star-forming disks at $z \approx 2$ are already relatively metal-rich (Erb et al. 2006; Queyrel et al. 2009). The spatial structure of the ISM and the star formation efficiency can be affected by metallicity, but only much lower metallicities (a tenth solar and below) would have a strong impact (Kraljic et al. 2014). The $\mathrm{CO}$ abundance in molecular gas also depends on metallicity, but for the average metallicity variations between $z=0$ and 2 for massive disk galaxies (less then a factor two), the metallicity effect on $\alpha_{\mathrm{CO}}$ remains modest (Leroy et al. 2011; Genzel et al. 2012) and eventually not stronger than the effect of galaxy type found in our present results.

The SB merger and spiral simulation exist at $1.5 \mathrm{pc}$ resolution (Renaud et al. 2014), but such extremely high spatial resolution is hard to reach for the high- $z$ disk model without lowering the mass resolution, because the higher gas mass in this case implies a larger number of resolution elements. For consistency we here analyze all models at 3 pc resolution, knowing that the star formation rate history and density distribution (PDF) have been shown to converge with respect to resolution at this $3 \mathrm{pc}$ scale in Renaud et al. (2014).

It is known from previous analyses of these simulations (Bournaud et al. 2014; Renaud et al. 2014; Kraljic et al. 2014) that these models have a total star formation efficiency (or gas depletion timescale) in agreement with the observed SchmidtKennicutt relation(s) (Kraljic et al. 2014; Renaud et al. 2014) and that the high- $z$ disk models proceed giant clumps of gas and star formation from which intense outflows are launched (the giant clumps in these high- $z$ galaxies typically contain $20-35 \%$ of the gas mass, up to $\sim 50 \%$ of the SFR, and a few percentage points of the total stellar mass, Bournaud et al. 2014). It is also known that the ISM is more turbulent in both high- $z$ disks and SB mergers than in spirals, with typical turbulent velocity dispersions in the $20-50 \mathrm{~km} \mathrm{~s}^{-1}$ range for high- $z$ disks and mergers, compared to $5-10 \mathrm{~km} \mathrm{~s}^{-1}$ for spirals. In addition, turbulent motions have been shown to be compressive primarily in SB mergers compared to spirals and high- $z$ disks: this means that around density peaks, radial turbulent motions compressing (or disrupting) the density peak are favored over solenoidal motions curling around the density peaking, in the case of galaxy mergers (Renaud et al. 2014).

Each simulation was analyzed in different snapshots, regularly spaced in time for disks, and in the starbursting phases for the SB merger model, as also indicated in Fig. 1. For disks, each snapshot was analyzed under three lines of sight: one faceon orientation, and two projections inclined by 60 degrees. The SB merger snapshots were analyzed under three perpendicular lines of sight. In the following, the average result was used to compute the molecular line emission for each galaxy model, unless stated otherwise.

\subsection{LVG analysis}

The interstellar gas distribution in AMR simulations is described with cells of variable sizes. Each cell has size $\epsilon$ and contains gas at a density $\rho$, temperature $T$, velocity $\boldsymbol{v}$, metallicity $Z$, etc. In the present simulations, the cell size $\epsilon$ is in the $3-12$ pc range for gas denser than $10 \mathrm{~cm}^{-3}$, and coarser cells only correspond to low-density (atomic or ionized) gas.

The purpose of the LVG analysis is to estimate the flux emerging from each individual cell for any given $\operatorname{CO}(J-$ $J-1)$ transition, and assuming that the line of sight of the pseudo-observation is given by the unitary vector $\boldsymbol{u}$. In the
LVG framework, the raw emission is determined by the physical parameter of the gas in the cell, but the radiation can be re-absorbed by molecules in the same cell, i.e. in the immediate (parsec-scale) vicinity of the emitting regions. This local re-absorption depends on how rapidly the line frequency shifts along the line of sight: if strong velocity gradients are present, the line central frequency is shifted by a greater amount than the intrinsic linewidth over a short distance, and the gas becomes transparent to its own emission, while weak velocity gradients imply that gas can re-absorb its own emission over longer distances. To account for this, we compute the $\mathrm{d} V / \mathrm{d} r$ parameter, where $r$ is the position along the line-of-sight axis $\boldsymbol{u}$, given by $\mathrm{d} V / \mathrm{d} r=\sum_{i=1,2,3} u_{i} \partial V_{i} / \partial r_{i}$. The gradient $\partial V_{i} / \partial r_{i}$ is determined on each axis $i$ by comparing the velocity field $v$ over neighboring cells.

The simulations employ a temperature threshold at high densities to prevent artificial fragmentation effects (Teyssier et al. 2010, Sect. 2.1): the hydrodynamic equations are solved assuming a pressure that cannot be lesser than a value calculated to keep the Jeans length larger than four cells at the maximal refinement level. The corresponding "numerical" temperature thus cannot be lower than $50-100 \mathrm{~K}$ in our simulations. However, the extra numerical pressure can be considered as a subgrid resolved for non-thermal processes (such as small-scale turbulent motions and stellar feedback winds rather than thermal energy, e.g., Teyssier et al. 2010). The pressure floor is then subtracted during the analysis to obtain only the physical temperature, i.e., the amount of thermal energy heating the gas above the menial temperature floor. We thus resolve cold high-density components down to $10-20 \mathrm{~K}$, where the temperature represents the true thermal energy after subtracting the extra numerical components (see above). Cooling through rotational molecular transitions in the molecular phase is not included in our simulations, and yet a two-phase interstellar medium is produced with a density-temperature distribution (Perret et al. 2014, Fig. 1) that is consistent with more detailed simulations of the ISM (e.g., Kim et al. 2013).

The combination of the local gas physical parameters and the $\mathrm{d} V / \mathrm{d} r$ measurement of the local, small-scale opacity of the ISM to its own emission directly indicates the net flux that emerges in each $\mathrm{CO}(J-J-1)$ transition through the LVG tables from Weiß et al. (2005), which assume collision rates from Flower (2001) and a standard CO abundance (Frerking et al. 1982; Lacy et al. 1994). The underlying assumption in this modeling is that the $\mathrm{CO}$ emission is not re-absorbed on large scales, which are well resolved in our simulations $(\leq 10-100 \mathrm{pc})$, because the turbulent structure of the molecular ISM makes it unlikely to find two molecular clouds along the same line of sight and with a velocity difference small enough to allow an overlap of the $\mathrm{CO}$ emission/absorption lines with their intrinsic linewidth (a few $\mathrm{km} \mathrm{s}^{-1}$, lower than the velocity shifts induced by galactic rotation on large scales). The same technique can be used for other molecular transitions such as HCN lines, which are not studied in the present paper.

Some of the calculations presented hereafter require estimating the total molecular mass (i.e., the $\mathrm{H}_{2}$ mass). Our numerical simulations are performed at very high resolution, which has the advantage of explicitly resolving the dense gas clouds and their substructures, up to densities higher than $10^{6} \mathrm{~cm}^{-3}$, without requiring a sub-grid model to be assumed for the cloud mass function during the post-processing. This does, however, come at the expanse of including a molecule-formation model in the simulations themselves, as could be done at reasonable computer cost 


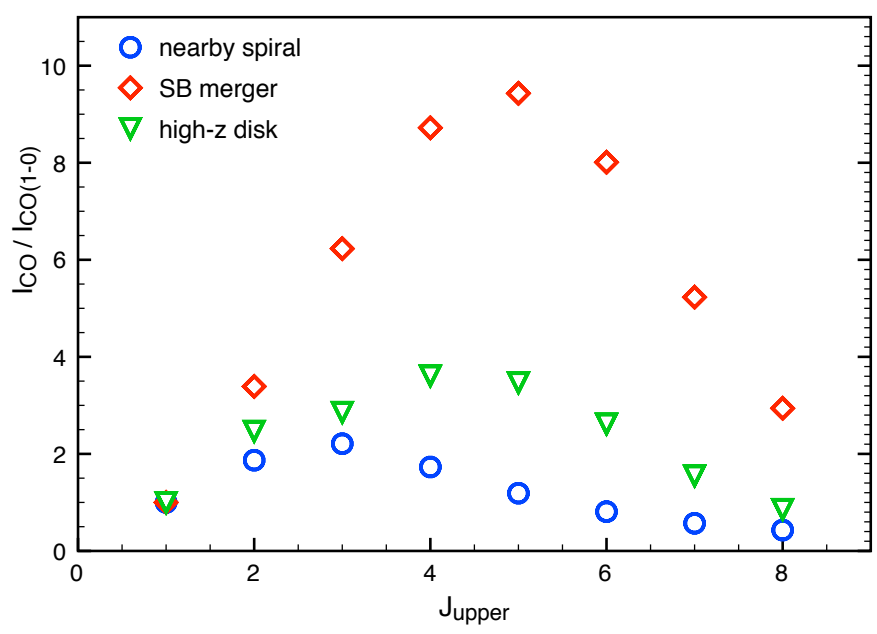

Fig. 2. Spectral line energy distributions (SLEDs) of the CO molecular for the three galaxy types, averaged over the different snapshots and projections analyzed for each galaxy type. The SLEDs are normalized to the $\mathrm{CO}(1-0)$ line intensity. See text for details on the fluctuations between snapshots and projections at a fixed galaxy type.

in simulations that employ more modest maximal resolutions (Narayanan et al. 2011; Feldmann et al. 2012a). When required, we hereafter assume that the ISM becomes molecular above a simple density threshold. We use two values for the threshold, of 10 and $50 \mathrm{~cm}^{-3}$, and keep the average result. These two values bracket the typical densities at which the ISM starts to be molecule-dominated in state-of-the-art models of entire galaxies with an accurate molecule formation scheme (e.g., Feldmann et al. 2012b). The fraction of the ISM mass encompassed in the $10-50 \mathrm{~cm}^{-3}$ range is small enough for the related uncertainty on the results to be limited, and smaller than case-to-case fluctuations for each type of galaxy (uncertainty below $10 \%$ for the $\left.\alpha_{\mathrm{CO}}\right)$.

\section{Results}

\subsection{CO spectral line energy distributions}

The CO spectral line energy distributions (SLED) are shown in Fig. 2 for each type of galaxy, averaged over the different snapshots and projections analyzed and normalized to the $\mathrm{CO}(1-0)$ line intensity. While there are strong differences with galaxy type, the differences between different snapshots or projections for a given galaxy type are relatively small. The rms individual variations of the $I_{\mathrm{CO}(2-1)} / I_{\mathrm{CO}(1-0)}$ over individual snapshots range from $12 \%$ for the spiral galaxy to $16 \%$ for the high $z$ disk and $32 \%$ for the SB merger. The rms variations of the $I_{\mathrm{CO}(5-4)} / I_{\mathrm{CO}(1-0)}$ range from $21 \%$ for the spiral galaxy to $27 \%$ for the high- $z$ disk and $56 \%$ for the SB merger. As a result, the differences in the average CO SLED between the various types of galaxies, as seen in Fig. 2, are much greater than the intrinsic variations at fixed galaxy type.

The intrinsic variations in the SLED are larger for the SB merger than for the spiral and high- $z$ disk, which can be attributed to the varying level in the starburst activity. All the selected snapshots for the SB merger correspond to phases where the SFR is elevated, but the exact level of SF activity varies, and the physical properties of the starbursting ISM also vary in this model. The starburst starts in a highly fragmented, spatiallyextended medium, and evolves toward a more concentrated nuclear starburst, thereby qualitatively recovering the observed variety of starbursting mergers (see Renaud et al. 2014, for details). Based on this, and given that the SB merger analyzed here

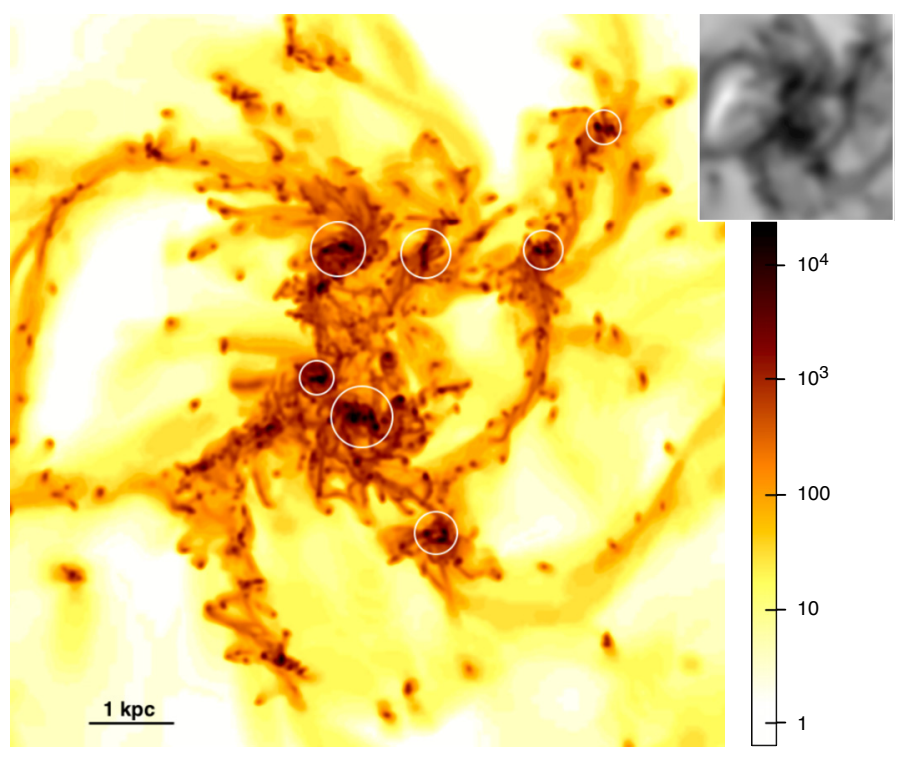

Fig. 3. Face-on gas density map of a snapshot of our high- $z$ disk model, used to analyze the contribution of "giant clumps" to the global CO SLED. The gray-scale inset displays a mock HST-like optical observation ( $B$-band rest-frame emission with a Gaussian PSF of FWHM $500 \mathrm{pc}$ ), which shows an irregular structure with a few giant clumps and some spiral arms, typical of real $z \approx 2$ star-forming galaxies (Elmegreen et al. 2007). The seven giant clumps (with stellar masses above $10^{8} M_{\odot}$ ) are circled on the gas map, with the clump radius computed as in Bournaud et al. (2014). These seven clumps contain about half of the total SFR and one third of the total gas mass. The SLEDs corresponding to the emission for the clump regions and from the rest of the galaxy are analyzed in Fig. 4. Gas number densities are in $\mathrm{cm}^{-3}$, and the map is smoothed at a $10 \mathrm{pc}$ scale.

is about the ILRG/ULIRG transition, its average SLED should be considered as representative of such systems, which are relatively common among major mergers. More extreme starbursts that are less common at least at low redshift could very likely show even greater excitation in their CO SLED properties.

The CO SLED of the spiral galaxy model is consistent with that of the Milky Way disk, and that of the SB merger model is consistent with observations of starbursting mergers about the LIRG/ULIRG activity levels (see Introduction). Our AMR simulations and LVG analysis thus yield realistic results for these two classes of galaxies, for which the typical SLED properties are relatively well constrained observationally - as opposed to high-redshift disks. As for the high-redshift disks, we find that the predicted CO SLED is generally closer to that of a spiral galaxy than of a starbursting merger, but with a significant excess of high-excitation components compared to spiral galaxies. The $I_{\mathrm{CO}(2-1)} / I_{\mathrm{CO}(1-0)}$ and $I_{\mathrm{CO}(3-2)} / I_{\mathrm{CO}(1-0)}$ ratios for the high- $z$ disk are moderately higher than for the spiral galaxy model, but these ratios become much greater than in the spiral galaxy model when CO transitions with $J_{\text {upper }} \geq 4$ are considered. Actually, the high- $z$ disk model presents a surprisingly high $I_{\mathrm{CO}(4-3)} / I_{\mathrm{CO}(3-2)}$ ratio, greater than its $I_{\mathrm{CO}(3-2)} / I_{\mathrm{CO}(2-1)}$ ratio, and this is a property that is not met in spiral disks and SB mergers. It is found in almost all snapshots (7 out of 8 ) of the high- $z$ disk model. The physical origin of the high-excitation components in high- $z$ disks compared to SB mergers will be analyzed later in Sect. 4 .

\subsection{Dissecting high-redshift clumpy disks}

To understand the origin of the high-excitation components in the CO SLED of high-redshift disks compared to nearby spirals, 


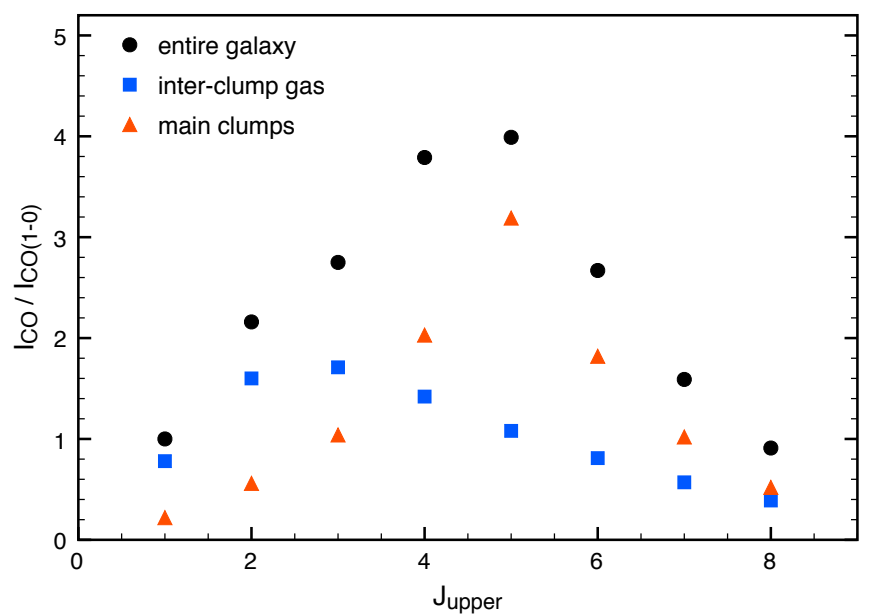

Fig. 4. CO SLEDs for the total galaxy, the seven giant clumps, and the rest of the gas on the high- $z$ disk snapshot shown in Fig. 3.

we performed a spatially resolved analysis of the representative snapshot shown in Fig. 3. This is the third snapshot in the time sequence of our high- $z$ disk simulation, so it has a gas fraction and clumpiness close to the average of the other snapshots, and it is representative of a typical $z \approx 2$ main sequence disk galaxy. We checked separately that the other snapshots show the same behavior in their spatially resolved properties, and here we show the results averaged over three perpendicular projections for the selected snapshots.

The chosen high-redshift disk snapshot has a clumpy and irregular morphology that is typical of high-redshift galaxies, and a gas fraction of $53 \%$ at the instant analyzed. We identified the seven most massive clumps and measured their optical radius as in Bournaud et al. (2014). These giant clumps have baryonic masses in the $1.7-7.3 \times 10^{8} M_{\odot}$ range and contribute to $57 \%$ of the total SFR of the galaxy. The interclump medium has a lower average density but does contain high-density clouds on small scales, with individual masses and sizes closer to that of low-redshift molecular clouds. This provides a significant component of relatively diffuse star formation (although clustered on small scales $<100 \mathrm{pc}$ ).

The CO SLEDs were measured separately for the main giant clumps (summed over the clumps) and for the rest of the galaxy, i.e. the interclump medium. The results are shown and compared to the entire galaxy in Fig. 4. The clumps gas has a high-excitation SLED with an intensity ratio peaking at the 5-4 transition, which is qualitatively similar to the starbursting merger type. In contrast, the SLED of the interclump medium peaks in the $\mathrm{CO}(3-2)$ line, and the relative line ratios are close to those measured for nearby spirals, with differences smaller than $20 \%$ for $\mathrm{CO}(2-1), \mathrm{CO}(3-2)$ and $\mathrm{CO}(4-3)$ intensities relative to $\mathrm{CO}(1-0)$. A moderate excess is found for higher- $J$ transitions (about $35 \%$ in intensity) compared to nearby spirals.

The global CO SLED of high- $z$ star-forming disk galaxies thus appears to result from the combination of a low-excitation, nearly Milky-Way-like component in the diffuse disk, containing about half of the total gas mass and one third of the star formation rate, and a higher-excitation component in a few giant clumps that contain two thirds of the total star formation rate. The origin of the high $\mathrm{CO}$ excitation there is analyzed in the next section. The combination of these two components results in the increased $\mathrm{CO}(4-3) / \mathrm{CO}(3-2)$ line intensity ratio compared to the $\mathrm{CO}(3-2) / \mathrm{CO}(2-1)$ one, a feature found in the high- $z$ snapshot

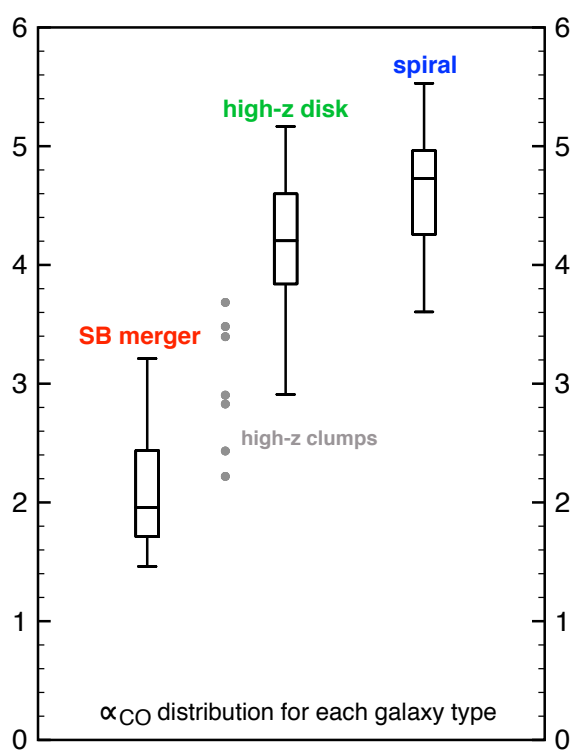

Fig. 5. Statistical distribution of the $\mathrm{CO}$ luminosity-to- $\mathrm{H}_{2}$ mass conversion factor $\left(\alpha_{\mathrm{CO}}\right)$ for each galaxy type. The symbols indicate the extreme values, quartiles, and median for each galaxy type. The statistical scatter is dominated by variations between snapshots and projections, the uncertainty on the molecular mass itself being a minor contribution to the scatter (see text). The gray dots show individual giant clumps in a representative high-redshift galaxy (see Sect. 3.2 and Fig. 3).

Table 2. Average value of the $\alpha_{\mathrm{CO}}$ conversion factor for each galaxy type, averaged over all snapshots and projections.

\begin{tabular}{lc}
\hline \hline Model & $\alpha_{\mathrm{CO}}$ range \\
\hline spiral & $4.3-4.7$ \\
high- $z$ disk & $3.9-4.5$ \\
SB merger & $1.7-2.2$ \\
\hline
\end{tabular}

Notes. The indicated range corresponds to the extreme assumptions for the molecular mass definition. $\alpha_{\mathrm{CO}}$ is in $M_{\odot}\left(\mathrm{K} \mathrm{km} \mathrm{s}^{-1} \mathrm{pc}^{2}\right)^{-1}$ throughout the manuscript.

analyzed here (Figs. 3, 4) and on average for high-z disk snapshots (Fig. 2).

\subsection{CO luminosity-to-molecular gas mass conversion factor}

The $\mathrm{CO}(1-0)$ line intensity measured in the various snapshots and projections is compared to the mass of molecular gas present in the simulations, derived with various assumptions detailed in Sect. 2. This yields a statistical distribution of the $\alpha_{\mathrm{CO}}$ conversion factor for each galaxy class, as displayed in Fig. 5. We summarize in Table 2 the average value of $\alpha_{\mathrm{CO}}$ for the highest and lowest assumptions on the molecular gas mass, for each galaxy type. Our extreme assumptions on the molecular gas mass definition yield an uncertainty of $6-10 \%$ on the average $\alpha_{\mathrm{CO}}$ for the various galaxy types. This is smaller than the variations between snapshots and projections, which induce an rms variation in the recovered $\alpha_{\mathrm{CO}}$ factor of $11 \%$ for spirals, $13 \%$ for high-redshift disks, and $22 \%$ for starbursting mergers.

The larger relative variations in $\alpha_{\mathrm{CO}}$ for SB mergers correspond to the natural variations in the SFR activity along the merger time sequence. We compare in Fig. 6 the median estimate of $\alpha_{\mathrm{CO}}$ to the SFR for individual snapshots of the SB merger model. There is a trend toward lower $\alpha_{\mathrm{CO}}$ values when the SFR is at its highest, i.e. about $80-100 M_{\odot} \mathrm{yr}^{-1}$. While our SB merger 

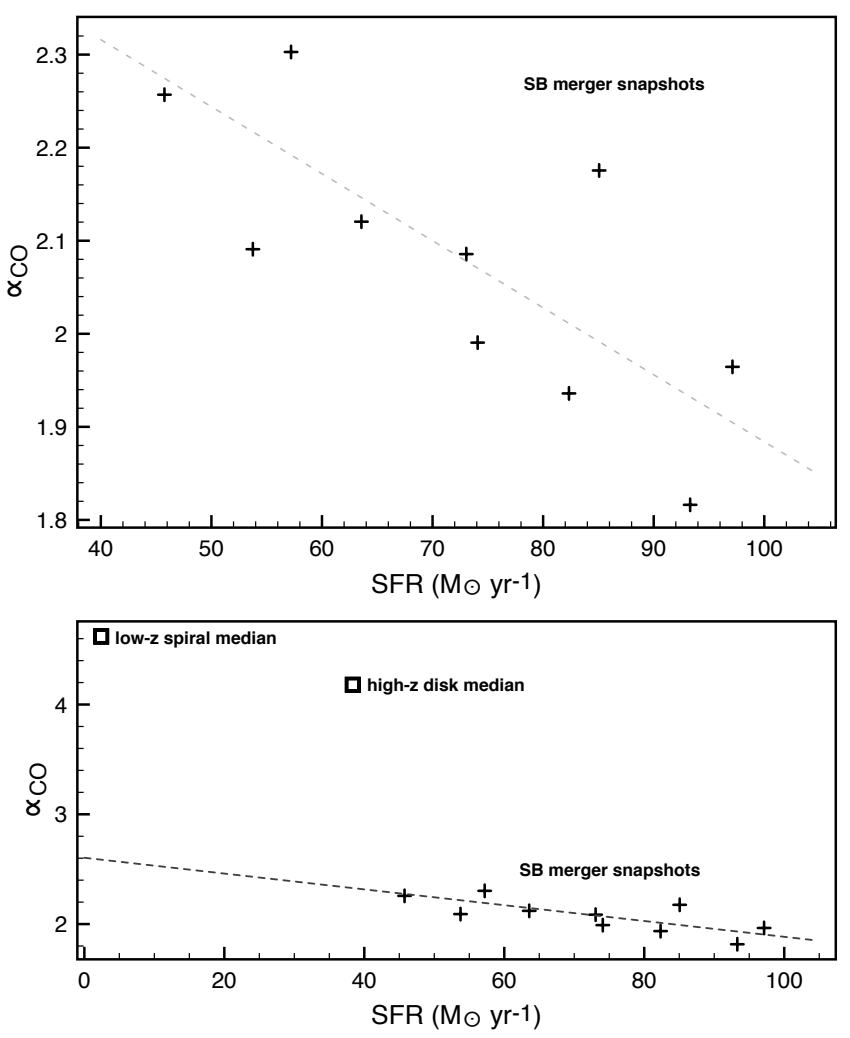

Fig. 6. Comparison of the retrieved $\alpha_{\mathrm{CO}}$ conversion factor to the SFR for each individual snapshot of the SB merger model (top - averaged over three projections for each snapshot). A clear correlation is found between SFR and $\alpha_{\mathrm{CO}}$ within the SB merger galaxy type, and the intrinsic variations in the SFR in the SB merger model induce a larger scatter in $\alpha_{\mathrm{CO}}$ than for isolated disk models. This indicates that stronger starbursts could have even lower $\alpha_{\mathrm{CO}}$ factors, given that the merger model used here is moderately starbursting about at the LIRG/ULIRG transition regime. In contrast, other galaxy types do not follow this $\alpha_{\mathrm{CO}}-\mathrm{SFR}$ trend retrieved for the SB merger snapshots (bottom). In particular, high- $z$ disks have an SFR that is almost as high as some SB merger snapshots, but with much higher $\alpha_{\mathrm{CO}}$ (see also Fig. 11).

is just reaching the level of ULIRGs, this suggests that more extreme merger-triggered starbursts most likely have even lower $\alpha_{\text {CO }}$ values (although higher SFR enhancements are rare, see, e.g., Di Matteo et al. 2007, so higher star formation rates in mergers may require different type of progenitor galaxies).

However, when considering the various systems in our sample (low- $z$ spiral, high- $z$ disk, SB merger), there is no uniform trend between the $\alpha_{\mathrm{CO}}$ factor and the SFR, and the disks show a systematic deviation from the trend retrieved between SB merger snapshots (Fig. 6 bottom). Actually, our high- $z$ disk model has an SFR close to the most moderate SB merger snapshots selected for our analysis, but has much higher $\alpha_{\mathrm{CO}}$. We show below that this relates to the different small-scale phase-space structures of the ISM. Such behavior by $\alpha_{\mathrm{CO}}$, which only weakly varies for main sequence galaxies independent of redshift and SFR but rapidly drops for starburst systems with an $\alpha_{\mathrm{co}}$-SFR correlation for the latter category, is consistent with the results of Sargent et al. (2014, Fig. 15b). (In detail, Sargent et al. find a somewhat stepper relation between $\alpha_{\mathrm{CO}}$ and SFR for starbursts, but with a broad scatter.)

We also show in Fig. 5 the $\alpha_{\mathrm{CO}}$ factor for the giant clumps identified in a representative snapshot of the high- $z$ disk model (the one used in Figs. 3, 4 and Sect. 3.2). These giant clumps

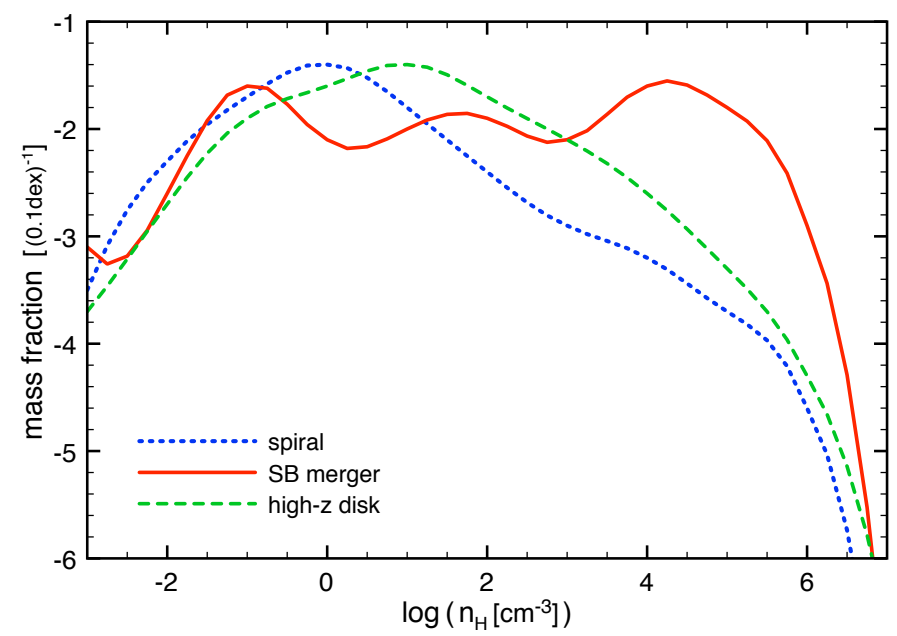

Fig. 7. Density point distribution function (PDF) of the gas for each galaxy type (averaged over the different snapshots). The local spiral and high- $z$ disk models have density PDFs that are well described by a log-normal functional shape, with a small excess of high-density gas in the form of a power-law tail, which corresponds to self-gravitating gas (Renaud et al. 2013; Bournaud et al. 2014). SB mergers strongly deviate for a log-normal PDF and have a strong excess of high-density gas. The rapid drop in the PDFs above $10^{6} \mathrm{~cm}^{-3}$ corresponds to the resolution limit of our simulations.

have individual $\alpha_{\mathrm{CO}}$ factors lower than their entire host galaxy, although not as low as the SB merger model. This appears consistent with the fact that these kpc-clumps behave like moderate starburst regions in the Schmidt-Kennicutt diagram; that is, they have gas depletion timescales shorter than their entire host galaxies, but not as short as the most intensely starbursting mergers (Freundlich et al. 2013, for observations; Zanella et al., in prep., for simulations). The low $\alpha_{\mathrm{CO}}$ factors of the clumps explain why the $\alpha_{\mathrm{CO}}$ of entire high- $z$ disks is somewhat lower than that of spirals: on average $23 \%$ of the $\mathrm{CO}(1-0)$ line intensity from the whole galaxy is emitted by the highly excited giant clumps, while the bulk of the $\mathrm{CO}(1-0)$ emission remains dominated by the low-excitation large-scale disk.

\section{Interpretation}

\subsection{Origin of the high-excitation components in starbursting mergers and high-redshift disks}

Here we analyze the origin of the strong excitation of high- $J$ $\mathrm{CO}$ lines further, first in SB mergers compared to spirals, then in high- $z$ disks (in particular their giant star-forming clumps). In particular, we aim at disentangling the role of high-density gas and warmed gas in the CO line properties of such systems.

\subsubsection{Starbursting mergers}

The average density PDF for our SB merger and spiral simulation snapshots are shown in Fig. 7. The SB merger shows a strong excess of high-density gas with densities above $10^{3} \mathrm{~cm}^{-3}$; the mass fraction of gas found at densities in the $10^{4}-10^{5} \mathrm{~cm}^{-3}$ range is more than ten times higher than in spirals. This highdensity gas excess is found in major merger simulations on various interaction orbits, once the numerical resolution is high enough (Teyssier et al. 2010; Bournaud et al. 2011; Powell et al. 2013). It arises from compressive regions in the tidal field of interacting galaxies, which are frequently found in early-stage 


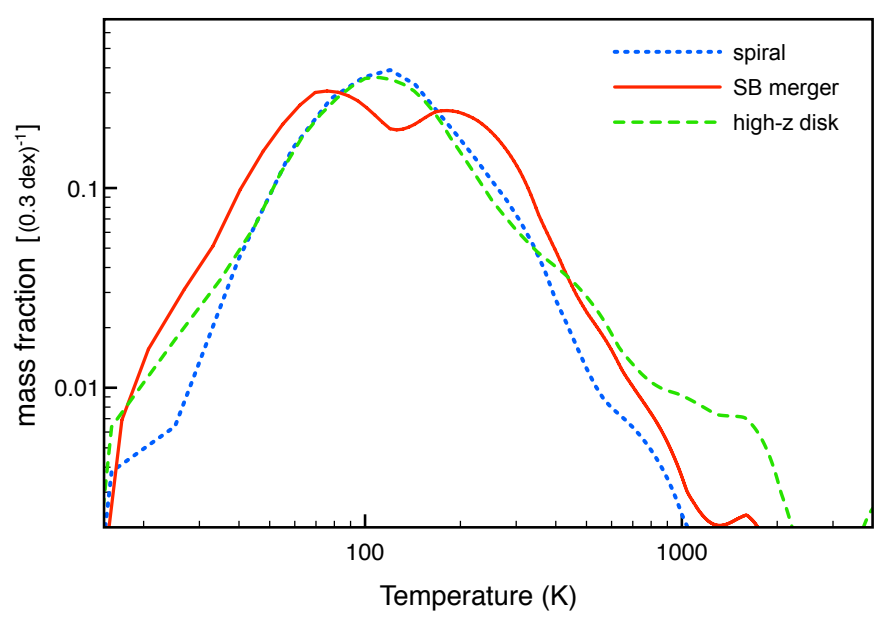

Fig. 8. Temperature PDF for the three types of galaxies, averaged over the selected snapshots. Gas denser than $100 \mathrm{~cm}^{-3}$ is considered here.

interactions, inducing an excess of compressive turbulent motions down to small scales $(10-100 \mathrm{pc})$ and persisting until the final galactic coalescence. Such excesses of high-density gas are also suggested from observations of high-density tracers in starbursting mergers (Gao \& Solomon 2004; García-Burillo et al. 2012).

Compared to this strong excess of high-density gas, there is no major excess of warm molecular gas in our SB mergers. The temperature PDF for molecular gas is shown in Fig. 8. Of course, increased stellar feedback from the triggered star formation activity does produce warmer gas in the SB merger model compared to spirals. However, this warm gas excess ${ }^{3}$ involves less than $7 \%$ of the total molecular gas mass: the mass of gas warmer than $100 \mathrm{~K}$ in the SB merger temperature PDF in excess of the spiral temperature PDF is $5.8 \%$ of the molecular gas mass. Actually, gas affected by stellar feedback in our SB merger simulation rapidly evolves into a low-density wind $\left(<10^{2-3} \mathrm{~cm}^{-3}\right)$ representing a small fraction of the ISM mass and not the molecular phase. As a result, the warm gas excess is much weaker than the excess of high-density gas independent of temperature.

To determine the contribution of high-density and diffuse gas on the overall CO SLED, we performed control experiments with matched density or temperature distributions between spirals and SB mergers. The $\mathrm{CO}(5-4) / \mathrm{CO}(1-0)$ line intensity ratio on the average CO SLED is 8.1 times higher in SB mergers than in spirals. When we reanalyzed the simulations assuming that all gas denser than $10^{2} \mathrm{~cm}^{-2}$ is isothermal at $300 \mathrm{~K}$ (for both the mergers and the spirals), this factor becomes 6.4 , so that the difference is still largely present without any temperature effect. Conversely, when we selected gas cells in the SB merger model with a probability computed to match the density PDF of the spiral snapshots (i.e., we kept most cells with densities of a few $10^{2} \mathrm{~cm}^{-3}$, but only a small fraction of the cells with the highest densities, to artificially "remove" the dense gas excess), without modifying the temperatures, the same ratio drops to 2.8 , showing that high $\mathrm{CO}$ excitation in SB mergers is much weaker with only a warm gas excess and no high-density gas excess.

The simulations clearly show, therefore, that the differences in the CO SLED between disks and SB mergers are mainly due to the excess of dense gas created by gas compression in the

\footnotetext{
3 Interestingly, the extra warmed gas in the SB mergers is not just from stellar feedback, but also from enhanced small-scale shock compression (Bournaud et al., in prep.).
}

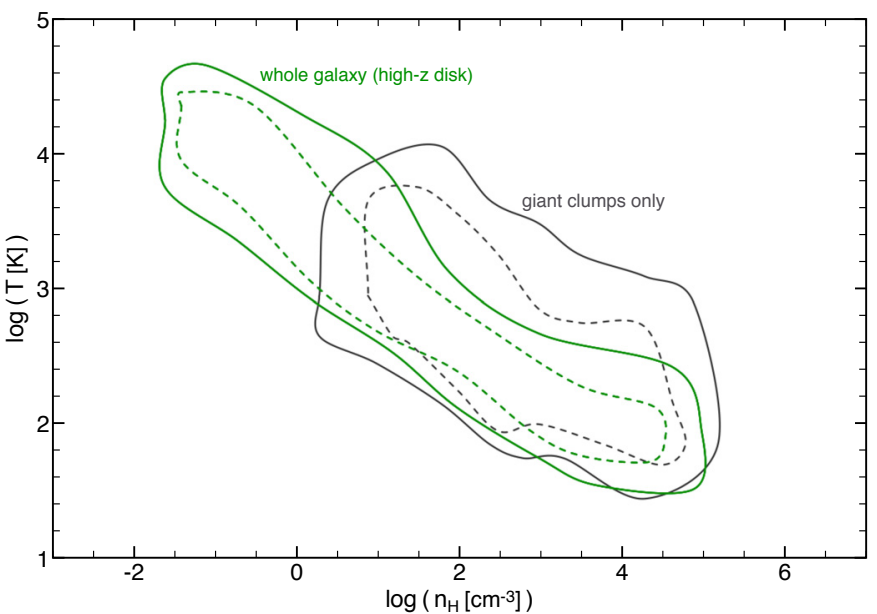

Fig. 9. Phase-space distribution of the ISM in a high- $z$ disk snapshot (black) and its giant clumps (green), selected as in Figs. 3 and 4. The figure shows the density-temperature space, with isodensity contours encompassing $90 \%$ of the total gas mass (solid) and 67\% of the total gas mass (dashed).

mergers. This gas compression results from both the large-scale gas inflows and the compressive properties of merger-induced turbulence on small scales (e.g., Renaud et al. 2014).

\subsubsection{High-redshift disks}

The phase space distribution of the interstellar gas in our high$z$ disk models (Figs. 7 and 8) shows a modest excess of highdensity gas compared to spirals, but in much weaker proportions than in SB mergers. There is also a small excess of warm gas, somewhat greater than in SB mergers, but encompassing a small fraction of the molecular gas mass: the excess of gas at $n_{\mathrm{H}}>10^{2} \mathrm{~cm}^{-3}$ at $T>10^{3} \mathrm{~K}$, compared to spirals, represents $2.8 \%$ of the mass of gas that is denser than $10^{2} \mathrm{~cm}^{-3}$. This is consistent with the fact that the average CO SLED of high- $z$ disks is close to that of low-redshift spirals, especially when compared to starbursting mergers: the excess of warmed gas remains quite limited, and the large excess of high-density gas present in SB mergers and explaining their high excitation is mostly absent in high-z disks.

Things change at the scale of star-forming clumps, which we analyzed using the same spatial selection as in Sect. 3.3 and Fig. 3. These giant clumps span the high-density regime of the global density distribution function, but do not induce a major excess of high-density gas as does the one observed in SB mergers. Indeed, typical numbers for a giant clumps in a redshift two-disk galaxy are a gaseous mass of $5 \times 10^{8} M_{\odot}$, an inplane half-mass radius of $300 \mathrm{pc}$, and a large vertical scale-height of $500 \mathrm{pc}$ or more (in both observations and hydrodynamic simulations, e.g. Bournaud et al. 2014, and references therein), yielding an average number density of $50-100 \mathrm{~cm}^{-3}$, so only marginally higher than that of the entire host galaxy.

However, the clumps do induce a significant excess of warmed gas at densities of 100 to $10^{5} \mathrm{~cm}^{-3}$. Indeed, the nonlinear dependence of star formation on gas density is such that star formation is more concentrated in the clumps than the gas mass itself (see Sect. 2), and the clumps gas is thus more exposed to stellar feedback per unit gas mass than is the interclump gas. A consequence is that galactic outflows are mostly launched by the giant clumps in our models (Bournaud et al. 2014; Perret et al. 2014; consistently with observations Genzel et al. 2011; and theory Dekel \& Krumholz 2013). This warm 


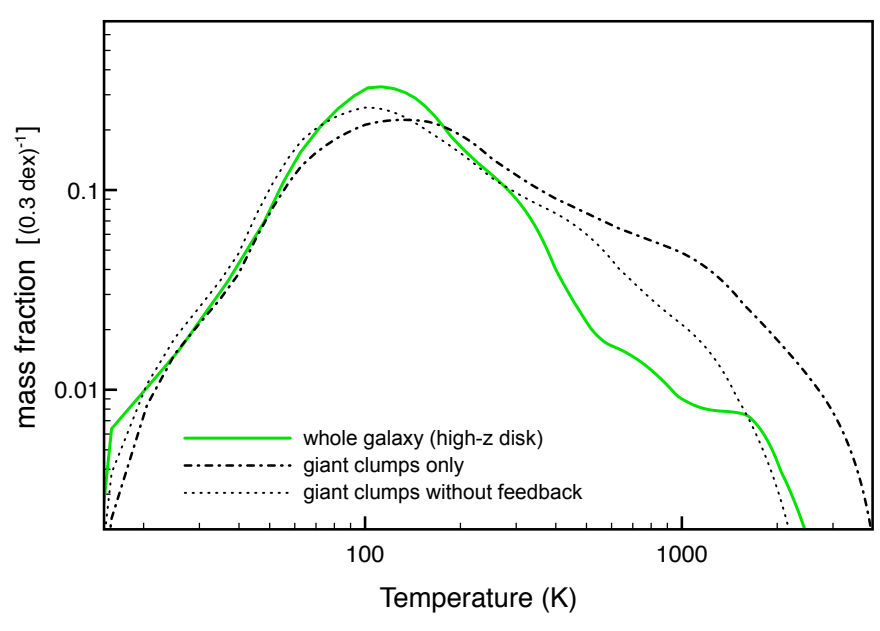

Fig. 10. Temperature PDF for the high- $z$ disk snapshot from Figs. 3 and 4 , showing the temperature distribution of gas in the $10^{3}-10^{4} \mathrm{~cm}^{-3}$ density range (i.e. moderately dense molecular gas). The thick green line is for the entire galaxy and thick dashed line for the giant clumps alone (selected as in Fig. 3), and the thin dashed line is for the giant clumps in the same simulation rerun without stellar feedback for $50 \mathrm{Myr}$ before the analyzed instant.

gas excess is illustrated by the phase-space distribution of the clumps' gas compared to that of the entire galaxies in Fig. 9. While less than $10 \%$ of the gas mass in the $100-1000 \mathrm{~cm}^{-3}$ density range is warmer than $10^{3} \mathrm{~K}$ in the entire high- $z$ galaxy, this fraction becomes as high as $23 \%$ in mass at the scale of the main giant clumps - it is below $5 \%$ in the interclump gas, and in lowredshift spirals.

We checked further that this warm gas excess is the main cause for the bumped CO SLED found for high- $z$ disks, by reanalyzing the simulation with a temperature capped at $300 \mathrm{~K}$ for any gas denser than $100 \mathrm{~cm}^{-3}$. This experiment reduces the $\mathrm{CO}(4-3)$ line intensity to $63 \%$ of its initial value for the entire galaxy (and to $39 \%$ of its initial value for the giant clump regions alone), bringing it close to Milky-Way-like excitation. This experiment also increased the typical $\alpha_{\mathrm{CO}}$ range from 3.9-4.5 to 4.0-4.7, again bringing it close to the values obtained for spiral disks.

There is thus a significant difference between the highexcitation components found in SB mergers and high- $z$ disks, regarding their physical origin. The highly excited CO SLED of SB mergers results mostly for the excess of high-density, rapidly star-forming gas among the molecular phase, which is the outcome of tidal compression and modified ISM turbulence (excess of compressive turbulent modes, Renaud et al. 2014). The moderately excited, bumped CO SLED and moderately lowered $\alpha_{\text {CO }}$ factors for high- $z$ disks results from gas heated by shocks and/or stellar feedback in the giant clumps of star formation that are ubiquitous in high-redshift star-forming galaxies, without involving a major excess of very high-density gas. We analyzed this further by examining the temperature PDF on the high- $z$ disk simulation (Fig. 10) for the entire galaxy, for the clumps alone, and for the same model evolved without stellar feedback during $50 \mathrm{Myr}$ before the analysis. We find that the warm gas excess is largely driven by stellar feedback in the high-SFR-density clumps, but that a substantial excess of warm gas would still be found in the clump regions compared to the other regions, this part at least being attributable to (turbulent) shocks and compression of gas in the clumps.
It is interesting to note that gas heating by stellar feedback behaves differently in the SB mergers and high- $z$ disks. These models have similar SFRs, so similar rates of energy injection through feedback. In SB mergers, less than $10 \%$ of the mass of gas denser than $100 \mathrm{~cm}^{-3}$ is significantly heated (see Sect. 3.4.1 and Fig. 8) and a larger fraction of the re-injected energy is rapidly found in thermal and kinetic form in a low-density wind (Bournaud et al., in prep.). In the high- $z$ disks, and in particular in their giant clumps, most of the feedback energy is injected in dense kpc-sized clumps and trapped in dense gas, resulting in a larger mass fraction of heated gas for densities higher than $100 \mathrm{~cm}^{-3}$ (about $30 \%$ of the dense gas mass inside the giant clumps is significantly heated, see Fig. 9). In spite of the warmed component in the high-excitation clumps, the bulk of the interstellar gas mass is only modestly heated compared to nearby galaxies (Fig. 8). This is consistent with the dust temperature estimates for main sequence galaxies at $z \approx 2$ by Magdis et al. (2012, see also Magnelli et al. 2014; Saintonge et al. 2013; and Genzel et al. 2014).

\subsection{Origin of the $\alpha_{C O}$ variations}

We have shown above how the excess of high-density gas in $\mathrm{SB}$ mergers can explain the excitation of high- $J$ CO lines. Nevertheless, such dense gas excess does not necessarily explain the lowered $\alpha_{\mathrm{CO}}$ found in these systems. The $\mathrm{CO}(1-0)$ emission is largely thermalized ${ }^{4}$ so that increased gas density cannot yield increased line temperatures at fixed $\mathrm{H}_{2}$ mass. Gas warmed by feedback effect may play a role, too. To probe this potential effect, we again use the control experiment in which the SB merger snapshots and the spiral ones have matched temperatures, as described in Sect. 4.1.1. The difference between the $\alpha_{\mathrm{CO}}$ factors of SB mergers and those of spirals is reduced only by $26 \%$, suggesting only a moderate contribution of the temperature effects in the variations of $\alpha_{\mathrm{CO}}$ from disks to SB mergers.

A major difference in the ISM of SB mergers is that it is much more turbulent than in progenitor spiral disks. In our models, we find that the mass-weighted average of the $1 \mathrm{D}$ velocity dispersion $\langle\sigma\rangle$ is 4.6 times higher in SB mergers than in spirals (here measured for densities in the $300-3000 \mathrm{~cm}^{-3}$ range to avoid any dependence on density). This is consistent with many merger simulations (Bournaud 2010, and references therein) and observations (Irwin 1994; Elmegreen et al. 1995; Duc et al. 2000; Wei et al. 2012). In addition, the turbulent velocity field in mergers shows an excess of compressive (curl-free) motions compared to solenoidal (divergence-free) motions that dominate the ISM in spirals - this outcome of the tidal galaxy interaction was presented in detail in Renaud et al. (2014). As a result, along a line of sight that crosses a dense gas cloud, the turbulent motions are more likely to be oriented radially from the cloud, so along the line of sight, rather than curling around the cloud perpendicular to the line of sight: this radial compression is the source of the high-density gas excess in mergers. Indeed we measure that the $\mathrm{d} V / \mathrm{d} r$ velocity gradient along lines of sight crossing gas denser than $300 \mathrm{~cm}^{-3}$ is 7.6 times higher in SB mergers than in isolated spirals, which is a factor that is even larger than for the bulk velocity dispersion. The CO linewidths emitted by such regions in SB mergers are broadened by similar factors, and at the same time, the line temperatures are affected in weaker proportions. For instance, at a density of $4000 \mathrm{~cm}^{-3}$

\footnotetext{
4 A large fraction of the $\mathrm{CO}$ is at moderate densities about $100 \mathrm{~cm}^{-3}$ but the $\mathrm{CO}(1-0)$ emission emerging from the galaxies is dominated at $73 \%$ by gas denser than $1000 \mathrm{~cm}^{-3}$.
} 
and at $50 \mathrm{~K}$, gas in spirals has a mean $\mathrm{d} V / \mathrm{d} r$ of $0.72 \mathrm{~km} \mathrm{~s}^{-1} \mathrm{pc}^{-1}$ corresponding to a $\mathrm{CO}(1-0)$ line temperature of $42.1 \mathrm{~K}$ in our LVG tables. The same gas in SB mergers has a mean $\mathrm{d} V / \mathrm{d} r$ of $5.1 \mathrm{~km} \mathrm{~s}^{-1} \mathrm{pc}^{-1}$ corresponding to a $\mathrm{CO}(1-0)$ line temperature of $34.3 \mathrm{~K}$, and, simultaneously, a 7.1 times broader linewidth, resulting in a $\mathrm{CO}(1-0)$ intensity that is more than five times higher per gas mass in this representative regime. As a result, the properties of ISM turbulence, modified in SB mergers compared to isolated spirals, cause a large broadening of emission lines, especially on lines of sight emanating from dense clouds, thereby increasing the intensity even when the line is thermalized and lowering the $\alpha_{\mathrm{CO}}$ conversion factor.

The behavior of high-redshift disks is different. Like mergers, they are highly turbulent systems, because of the gravitational instability and stirring by their giant clumps, but the highvelocity dispersions are mostly found in the giant clumps: we measure an increase in the mass-weighted velocity dispersion of a factor 4.7 in the giant clumps selected in Fig. 5 (compared to spirals), but only a factor 1.9 for the rest of the galaxy. The clumps dominate the high-excitation components, but make a limited contribution to the $\mathrm{CO}(1-0)$ line intensity. Furthermore, in the case of high-redshift disks, there is no preferential triggering of compressive motions, as opposed to SB mergers. In this case, we measure that the mean $\mathrm{d} V / \mathrm{d} r$ velocity gradient is 2.1 times higher in high- $z$ disks compared to spirals. The potential decrease in $\alpha_{\mathrm{CO}}$ is thus much more moderate than in SB mergers. At the same time, these systems are more gas rich than nearby mergers with higher mean densities (Fig. 7), which can lower the line temperature. The decrease in $\alpha_{\mathrm{CO}}$ is thus much more modest than in nearby SB mergers.

Grain photoelectric heating from UV star light and cosmic rays can be important heating processes in the low-excitation CO regions (e.g., Lequeux 2005). Cosmic rays are neglected in our simulations, and UV stellar light heating is also neglected in molecular regions. Photoionization by stellar photons is included in the ionized HII regions (Renaud et al. 2013), and given that most HII regions are close to the resolution limit of our simulation, the nearby molecular gas is numerically heated by thermal diffusion, providing a crude model of stellar feedback heating. An explicit modeling of the heating process would probably not yield more accurate results, since the stellar photons would generally be absorbed within the numerical resolution elements, especially in dense molecular regions. In any case, these processes depend mostly on the SFR, and since the SFE is almost similar in nearby spirals and high-redshift disks, the average heating rate per unit gas mass should be about similar, in contrast to turbulence dissipation, which largely increases from nearby spirals to high-redshift galaxies and starbursting mergers. In starbursting mergers, the extra heating from SFR-dependent processes could become more important per unit gas mass and boost the high-excitation components; nevertheless, in our model the extra heating in SB mergers generally seems modest (Fig. 8).

\subsection{The case of high-redshift mergers}

High-redshift mergers were not included in the present study. The main reason is the physical uncertainty in the typical properties of high-redshift mergers. While low-redshift simulations and high- $z$ disk ones have converged relatively well with resolution, feedback models, details of orbital parameters (Renaud et al. 2014; Bournaud et al., in prep.), and have an ISM structure very closed to observed ISM power spectra (Bournaud et al. 2010; Combes et al. 2012), the situation remains very unclear for major mergers of high-redshift galaxies.
Models with supernovae feedback have found that they can trigger a strong elevation of the SFR, like low- $z$ mergers, starting at a higher SFR level because of the rich gas reservoirs. But more recent models with higher resolution and thorough stellar feedback schemes actually question the ability of high- $z$ mergers to trigger the SFR as easily and frequently as low-redshift events, because the presence of dense giant clumps in pre-merger galaxies may saturate the SFR somewhat in many cases (Perret et al. 2014). The situation is also unclear in observations, since the rate of mergers should be much higher at high redshift, but the fraction of starburst galaxies with a specific star formation rate much above the average appears to barely increase with redshift (Rodighiero et al. 2011; Sargent et al. 2012; Schreiber et al., in prep.). Additionally, very high SFRs in high-redshift systems may alter the excitation process if the level populations are affected by radiation pumping.

Another reason for not studying high-redshift mergers here is more technical: the number of resolution elements required in our AMR simulations increases when we move to high redshift and increase the total gas mass and/or model merging systems with an excess of high-density gas (requiring finer AMR cells). Combining these two situations in a high- $z$ merger model would be extremely costly and would force us to use a lower resolution for our entire study, while the representativity of such a model, even in terms of global SFR, would be largely uncertain.

\section{Discussion}

\subsection{Comparison of $\alpha_{\text {CO }}$ to various parameters and models}

Our high-resolution AMR simulations coupled to LVG modeling of the $\mathrm{CO}$ emission and radiative transfer made it possible to predict the typical CO SLED and $\alpha_{\mathrm{CO}}$ conversion factor for various galaxy types. One of the main finding is that the $\mathrm{CO}$ excitation and $\alpha_{\mathrm{CO}}$ factor are not tightly linked to the SFR of a galaxy (see Fig. 11). Although such a correlation does exist at fixed galaxy types (for instance between different snapshots of our SB merger model, see Sect. 3.2), the high- $z$ disks have SFRs that are about as high as some SB merger snapshots, but they have an $\alpha_{\mathrm{CO}}$ factor that is almost as high as low- $z$ spirals and much higher than SB mergers (i.e., LIRGS/ULIRGs). When a high SFR (such as $50-100 M_{\odot} \mathrm{yr}^{-1}$ ) results from a major merger/interaction in a brief starburst phase, the modified ISM turbulence properties result in a strong reduction of the average $\alpha_{\mathrm{CO}}$ by a large factor compared to Milky-Way-like spirals - here by a factor 2.5-3 but we note that we have mostly analyzed moderately starbursting phases, so that extreme SBs may well have stronger dense gas excesses and even lower $\alpha_{\mathrm{CO}}$ factors. In contrast, when a similarly high SFR in a galaxy results from a high gas fraction at high redshift with a long duty cycle of sustained star formation (Daddi et al. 2010b; Elbaz et al. 2011), the $\alpha_{\mathrm{CO}}$ factor is much more moderately affected.

Figure 11 compares the value of $\alpha_{\mathrm{CO}}$ for all types of galaxies to the SFR, the SFR surface density $\Sigma_{\mathrm{SFR}}$, and the gas depletion time $t_{\text {dep }}=M_{\text {gas }} / S F R=1 / \mathrm{SFE}$. The last two quantities tend to show a better correlation with $\alpha_{\mathrm{CO}}$ than SFR, but still show strong systematic deviations for at least one galaxy type compared to the two others, showing that the dependence on galaxy type found in our work cannot be rendered by such global parameters. In particular, extrapolating a trend with $t_{\text {dep }}$ or $\mathrm{SFE}=1 / t_{\mathrm{dep}}$ from $\mathrm{SN}$ mergers to low- $z$ spirals would lead to underestimating the typical value of $\alpha_{\mathrm{CO}}$ for high- $z$ disks by about $30 \%\left(\alpha_{\mathrm{CO}} \approx\right.$ 3.1 instead of 4.3). Modeling the excitation of $\mathrm{CO}$ based on the global SFE, as done for instance in Papadopoulos et al. (2012), could thus overestimate the $\mathrm{CO}$ excitation in high- $z$ star-forming 

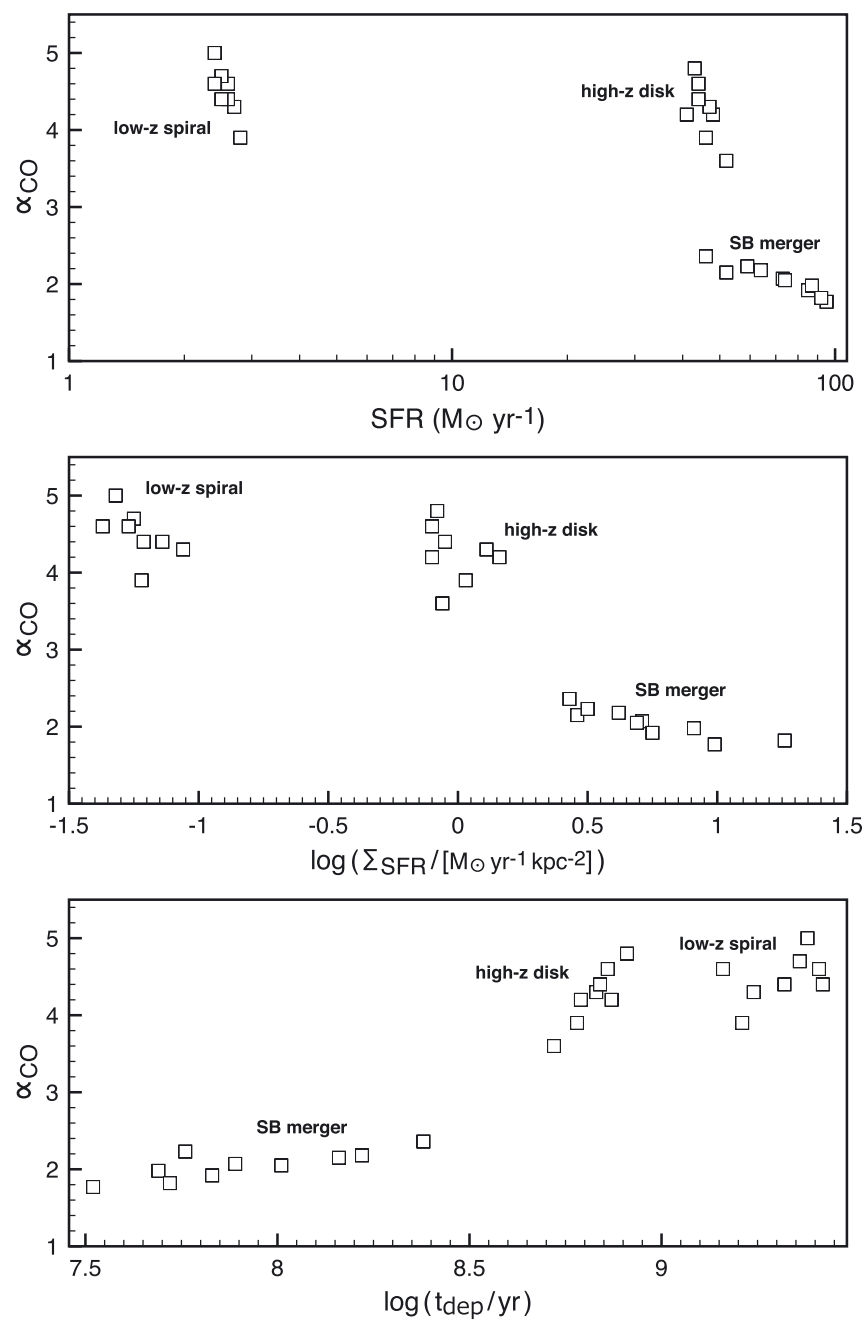

Fig. 11. Comparison of the $\alpha_{\mathrm{CO}}$ conversion factor estimated for each snapshot of each galaxy type (averaged over three projections and two assumptions for the molecular $\mathrm{H}_{2}$ mass in each case) to the absolute SFR (top), SFR surface density (middle) and gas depletion time (gas mass divided by SFR, bottom).

galaxies. As we have shown, excitation of $\mathrm{CO}$ by stellar heating and feedback mechanism is only significant in the giant clumps of high- $z$ galaxies, leaving the other half of the gas mass about unaffected.

We observe a better correlation between $\alpha_{\mathrm{CO}}$ and $\Sigma_{\mathrm{SFR}}$ in Fig. 11, although here again interpolating a power-law relation between spirals and mergers would underestimate $\alpha_{\mathrm{CO}}$ in high$z$ star-forming galaxies by about 20 percent. In this sense, our results are in relatively good agreement with the model proposed recently by Narayanan \& Krumholz (2014). The SFR density is, however, not the main common driver of the observed trend: the modest lowering of $\alpha_{\mathrm{CO}}$ in high-z disks does result from high gas excitation in regions of high SFR densities (giant clumps) and relatively large turbulent motions, but the large lowering of $\alpha_{\text {CO }}$ in SB mergers mostly results from large line widths in a highly turbulent ISM with ample velocity gradients $\mathrm{d} V / \mathrm{d} r$.

The retrieved ranges for $\alpha_{\mathrm{CO}}$ values in the three types of galaxies considered here agree with those derived from the dynamical arguments by Daddi et al. (2010b). Our models thus lend further support to the use of different $\alpha_{\mathrm{CO}}$ factors for disks and for SB mergers, even when the disks have high SFRs at high redshift, and of the resulting offset of starbursts in the SchmidtKennicutt plane (Daddi et al. 2010; Genzel et al. 2010; see also

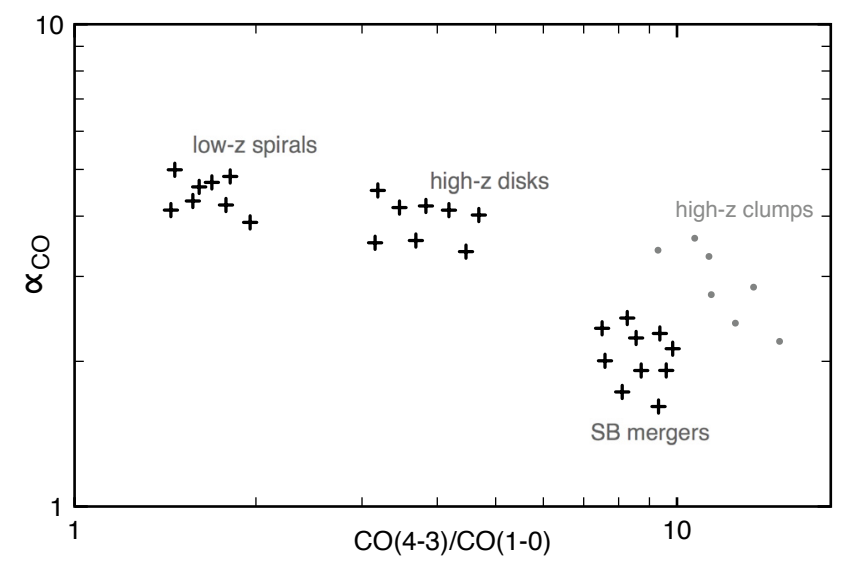

Fig. 12. Comparison of the $\mathrm{CO}(4-3) / \mathrm{CO}(1-0)$ line intensity ratio to the $\alpha_{\mathrm{CO}}$ conversion factor for each snapshot of each galaxy type (averaged over three projections and two assumptions for the molecular $\mathrm{H}_{2}$ mass in each case), showing that excitation is not necessarily a good discriminator for $\alpha_{\mathrm{CO}}$. Giant clumps identified in a high- $z$ disk snapshot are also shown (dots).

Saintonge et al. 2012). The theoretical interpretation proposed here is self-consistent, because the dense gas excess in mergers compared to gas-rich disks justifies the use of different $\alpha_{\mathrm{CO}}$ conversion factors, and at the same time this dense gas excess is theoretically expected to change the location of SB mergers in the Schmidt-Kennicutt diagram by lowering their gas consumption timescale by about 1 dex at a fixed gas surface density (Renaud et al. 2012).

There are of course other possible sources of variations in the $\alpha_{\mathrm{CO}}$ factor, even at fixed galaxy type. A key one on galactic scales is probably the gas phase metallicity (Israel 1997; Genzel et al. 2012). Here we have neglected these effects, assuming solar metallicity for all our galaxies of several $10^{10} M_{\odot}$. Variations with redshift up to at least $z=1-2$ (Erb et al. 2006; Queyrel et al. 2009; Wuyts et al. 2014) remain limited in this mass range: when compared to the Genzel et al. (2012) relations, they may only induce variations in $\alpha_{\mathrm{CO}}$ that remain smaller than those found in the present work with galaxy type. Similarly, the plausible dilution of the gas metallicity in mergers (Ellison et al. 2011; Di Matteo et al. 2007; Queyrel et al. 2012) is typically too small to dominate the changes in $\alpha_{\mathrm{CO}}$ with galaxy types found in our work. Metallicity-induced variations of $\alpha_{\mathrm{CO}}$ may dominate the effect studied here for much lower mass galaxies $\left(<10^{10} M_{\odot}\right)$ or at even higher redshifts $(z>2-3)$.

\subsection{Is excitation a good discriminator of $\alpha_{\mathrm{CO}}$ ?}

Figure 12 shows the $\mathrm{CO}(4-3) / \mathrm{CO}(1-0)$ intensity ratio and $\alpha_{\mathrm{CO}}$ conversion factor for each model snapshot. Although the highest excitation systems have the lowest $\alpha_{\mathrm{CO}}$ conversion factors, the correlation is very poor, not just because of large fluctuations but also because of systematic effects. In particular, high-redshift star-forming galaxies have high $\mathrm{CO}(4-3)$ luminosities coming from the highly excited components in their giant clumps, and these giant clumps represent only a limited fraction of the bulk gas mass, and only moderately lower the $\alpha_{\mathrm{CO}}$ factor of their whole host galaxies. Similar properties are found for $\mathrm{CO}(5-4) / \mathrm{CO}(1-0)$ and, in weaker proportions, for $\mathrm{CO}(3-2) / \mathrm{CO}(1-0)$. Our results thus indicate that $\mathrm{CO}$ excitation is probably not a good discriminator for the $\alpha_{\mathrm{CO}}$ conversion factor, especially when different types of galaxies are considered 


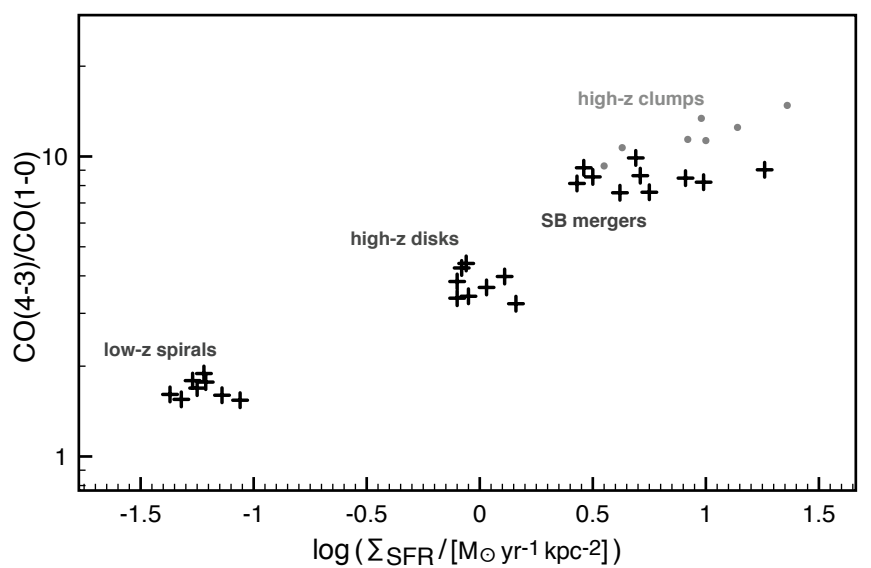

Fig. 13. Comparison of the $\mathrm{CO}(4-3) / \mathrm{CO}(1-0)$ line intensity ratio to the surface density of the SFR, showing a much tighter correlation of CO excitation with SFR density and that of $\alpha_{\mathrm{CO}}$ with SFR density (Fig. 11). Giant clumps identified in a high- $z$ disk snapshot are also shown (dots).

for the same amount of star formation (i.e., high- $z$ disks versus starburst mergers). The situation degenerates even further when high-redshift giant clumps are considered (Fig. 12) because their own $\mathrm{CO}$ excitation is even higher than that of SB merger, but their $\alpha_{\mathrm{CO}}$ factor remains larger. These giant clumps have high gas densities and they are highly turbulent, but they have $\mathrm{d} V / \mathrm{d} r$ radial velocity gradients that are lower than mergers.

The behavior of $\mathrm{CO}$ excitation across galaxy types and redshifts indeed appears to be different from the variations in $\alpha_{\mathrm{CO}}$. As shown in Fig. 13, CO excitation is well correlated to the surface density of the SFR, with little systematic deviations for high- $z$ disks - in contrast to the large systematics found when comparing $\alpha_{\mathrm{CO}}$ to the surface density of the SFR (Fig. 11, middle panel). Even the individual giant clumps inside high- $z$ disks follow the tight trend between SFR surface density and $\alpha_{\mathrm{CO}}$ in Fig. 13.

\subsection{Can $\alpha_{\text {CO }}$ be tightly linked to ISM turbulence?}

Our simulations and analysis have shown that the dependence of $\alpha_{\mathrm{CO}}$ on galaxy type is largely driven by the CO large line-widths in turbulent media. This could lead to the idea that measuring the turbulence of a given galaxy's ISM could be used to estimate its $\alpha_{\mathrm{CO}}$. This would actually be largely inaccurate. As shown in Fig. 14 there is only a broad trend for lower $\alpha_{\mathrm{CO}}$ at high ISM turbulent velocities $\sigma$, and the clumps of high- $z$ galaxies are almost as turbulent as SB mergers but their $\alpha_{\mathrm{CO}}$ factors remain almost $60 \%$ higher. The ISM turbulence in general is therefore not a tight tracer of $\alpha_{\mathrm{CO}}$ variations, especially when the bulk ISM turbulence $\sigma$ is measured (i.e., the rms velocity dispersion along a given line of sight or in three dimensions). The bulk turbulent speed in our high- $z$ disk models, about $20 \mathrm{~km} \mathrm{~s}^{-1}$, is very consistent with what is typically observed in $z=2$ star-forming galaxies (e.g., Tacconi et al. 2010 - the dispersions are higher in specific locations, such as inside the giant clumps and/or in the ionized gas phase, e.g., Förster Schreiber et al. 2011).

A much tighter correlation is found between $\alpha_{\mathrm{CO}}$ and the mean $\mathrm{d} V / \mathrm{d} r$ parameter of the various physical systems (Fig. 14 right). Indeed this parameter controls the CO line width more directly, because it indicates how rapidly the CO lines get Doppler-shifted along a given line of sight through radial velocity gradients around high-density peaks. A high bulk velocity dispersion $\sigma$ does not necessarily enlarge the CO lines, for
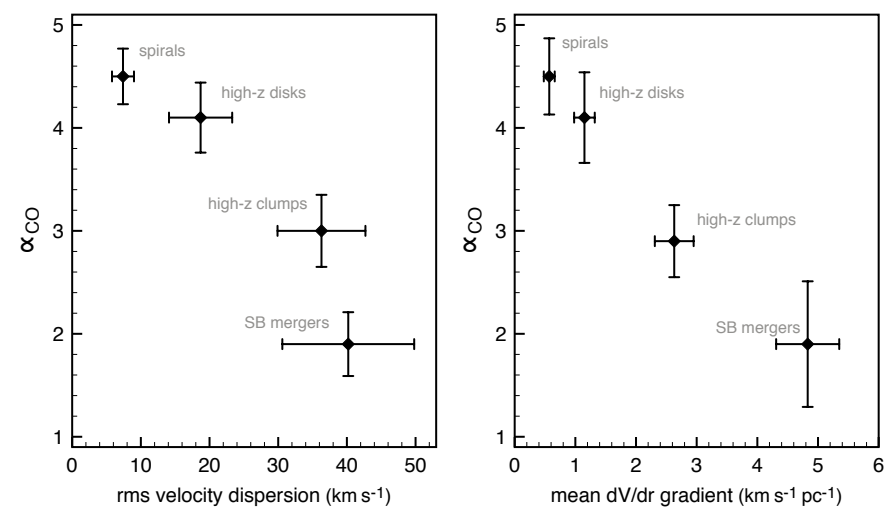

Fig. 14. Comparison of the $\alpha_{\mathrm{CO}}$ conversion factor to the bulk of ISM turbulence (left: rms 3D velocity dispersion divided by $\sqrt{(3)}$, massweighted average of the measurements for gas identified as molecular) and to the velocity gradient along lines of sight of molecular emission (right: mass-weighted average of the $\mathrm{d} V / \mathrm{d} r$ gradient along lines-of-sight crossing gas identified as molecular). Average values and rms scatter are displayed for each galaxy type and for the giant clumps of high- $z$ galaxies.

instance if gas is mostly in rotational motions around the molecular density peaks (as is often found in simulations of high- $z$ giant clumps, Ceverino et al. 2012): in such cases the radial gradients $\mathrm{d} V / \mathrm{d} r$ increase more slowly than $\sigma$. Starbursting mergers have the remarkable property of favoring the compressive turbulent motions, radially oriented around density peaks, so for the same turbulent speeds $\sigma$, the $\mathrm{d} V / \mathrm{d} r$ gradients get larger and the lowering effect on $\alpha_{\mathrm{CO}}$ is maximized.

Qualitatively, our results can, however, suggest that if galaxies continue to become increasingly turbulent at higher redshift, beyond what is observed at redshift two in recent surveys, the $\alpha_{\mathrm{CO}}$ parameters may be reduced.

\subsection{Comparison to real high-redshift star-forming galaxies: total gas fraction, conversion factor, and high-excitation components}

A noticeable prediction of this modeling is the presence of high-excitation components in the giant star-forming clumps of high-redshift galaxies, which weakly impacts the $\mathrm{CO}(1-0)$ and $(2-1)$ transitions and the associated conversion factors, but is more prominent for higher- $J$ transitions, resulting in an atypical CO SLED. Although high-excitation clumps contain only a limited fraction of the total gas mass of high- $z$ disks, our models predict that the effect could be noticeable in integrated observations of the high- $J$ lines of high-redshift gas-rich clumpy disks. We would like to point out that we have analyzed several snapshots of a single, very high-resolution simulation of a given galaxy, without exploring the variations in the clumpiness intrinsically present in high-redshift galaxies (perhaps induced by variations in the gas fraction, e.g. Salmi et al. 2012): this could induce substantial variations of the intensity of highexcitation components induced by the clump, while our representative model indicates that the $\mathrm{CO}(4-3)$ and $\mathrm{CO}(5-4)$ emission lines should generally be dominated by the highly excited clump components. Indeed, Weiß et al. (2005) have shown observationally in M82 that a high-excitation component is coming from star-forming clumps, along with a low-excitation component from the diffuse disk. The analogy lends support to the idea that the importance of high-excitation components in 
high-z star-forming galaxies could vary from galaxy to galaxy depending on their intrinsic clumpiness.

Our model predictions are consistent with the first observations of high-excitation $\left(J_{\text {upper }} \geq 4\right) \mathrm{CO}$ lines in main sequence star-forming galaxies about redshift two by (Daddi et al. 2014). They find that the $\mathrm{CO}(5-4)$ lines are brighter than expected from the $\mathrm{CO}(3-2) / \mathrm{CO}(2-1)$ line ratio. In more detail, these authors find that their observed data can be fitted well by a double-component CO SLED, with the low-excitation and high-excitation component taken from our high- $z$ disk simulation as being the clump gas and interclump gas (as shown in Figs. 5 and 6). They nevertheless find that in the majority of their systems (3 out of 4 ), the ratio of the high-excitation component to the low-excitation one has to be somewhat increased compared to our model (and unchanged for the 4th case). The best-fitting high-excitation fraction is correlated to the clumpiness. This is consistent with the idea above that we have modeled one high- $z$ galaxy with representative giant clumps formed by violent instability in a gas-rich disk, but also a specific degree of clumpiness in detail that is representative of a specific and somewhat arbitrary gas fraction, which does not cover the natural variations in this parameter.

The gas fraction in our models (and the resulting clumpiness) may be somewhat underestimated compared to real $z=2$ galaxies. It typically contains a $50 \%$ total gas fraction (slowly varying with time), counting all gas phases including the atomic one. The resulting density PDF (Fig. 7) actually shows that a substantial gas fraction lies in low-density phases below $10 \mathrm{~cm}^{-3}$ : this phase contains on average $26 \%$ of the total gas mass, i.e. $12 \%$ of the baryons, while $74 \%$ of the total gas (i.e. $38 \%$ of the baryons) would be in the molecular one. The density distribution of the turbulent ISM indeed preserves a substantial amount of gas in moderate-density region even if the average density of the system is high enough to form molecules. This suggests that our high- $z$ disk model has a realistic gas fraction, but on the rather gas-poor end compared to observations, hence that the high-excitation clumps could be somewhat more prominent in average galaxies. Conversely, if real galaxies at redshift two have a molecular gas fraction of 50\% compared to the stellar mass (i.e., molecular gas mass about equal to the stellar mass - Daddi et al. 2010; Genzel et al. 2010, 2014; see also Magdis et al. 2012) but also one fourth of their total gas mass atomic as suggested by our model, then their total gas fraction would actually be close to $60 \%$. In any case, the galaxy from the Daddi et al. (2014) sample with the closest clumpiness to our present high- $z$ disk model has a quantitatively very similar CO SLED.

\section{Conclusion}

We have used hydrodynamic simulations of low-redshift spirals, starbursting (SB) mergers, and high- $z$ disks with stellar masses about $6 \times 10^{10} M_{\odot}$ and a very high spatial resolution ( $3 \mathrm{pc}$ ), making it possible to finely resolve the interstellar medium structure and its interaction with a thorough set of stellar feedback sources (photo-ionization, radiation pressure, and supernovae). To model the molecular line emission from the $\mathrm{CO}$ molecule, we post-processed the simulations using a set of large velocity gradient grids, accounting for the local, small-scale excitation and for emission of molecular transition, as well as local opacity effects that depend on the phase-space distribution of the gas.

Our results highlight a strong dependence of the CO lines properties (CO SLEDs and $\alpha_{\mathrm{CO}}$ conversion factor) on galaxy type, in addition to other known dependencies (e.g., on metallicity). We found that:

1. Our model correctly reproduces the CO SLED properties of nearby spirals and SB mergers from the $\mathrm{CO}(1-0)$ transition to the $\mathrm{CO}(8-7)$ one, at least, and the high $\alpha_{\mathrm{CO}}$ factors estimated for the Milky Way. The absence of an explicit $\mathrm{H}_{2}$ formation model in the simulations only introduces uncertainties that are smaller than the intrinsic fluctuations of the $\mathrm{CO}$ line luminosities and of $\alpha_{\mathrm{CO}}$ for any given galaxy type.

2. SB mergers are characterized by strong turbulent compression of their ISM. This creates an excess of high-density gas among the cold molecular phase, which drives high excitation of CO. The excess of heating from the feedback and turbulence associated to the starburst plays a smaller role.

3. The CO SLEDs of high- $z$ star-forming galaxies are predicted be "bumped" around the $J_{\text {upper }}=4$ transition: namely, the $\mathrm{CO}(4-3) / \mathrm{CO}(3-2)$ and $\mathrm{CO}(5-4) / \mathrm{CO}(3-2)$ line intensity ratios can be as large or larger than the $\mathrm{CO}(3-2) / \mathrm{CO}(2-1)$ ratio, which is found for no other type of galaxy.

4. The main source of the high-excitation components in high- $z$ disks is an excess of warmed dense gas, found mostly in the giant star-forming clumps that are prone to shock and feedback heating, rather than an excess of high-density gas independent of temperature as in mergers. Given its origin in giant star-forming clumps, this high-excitation component should be stronger in clumpier galaxies.

5. Strong ISM turbulence in SB mergers generates broad COline widths with high line intensities and low $\alpha_{\mathrm{CO}}$ factors, around three times lower than spirals. The lowest values of $\alpha_{\mathrm{CO}}$ are found for the most intensely starbursting phases.

6. high- $z$ disks have $\alpha_{\mathrm{CO}}$ factors that are somewhat lower than the Milky-Way-like values that characterize nearby spiral models, but much closer to these than to SB mergers. The giant star-forming clumps, which are the high-excitation sites in high- $z$ disks, have $\alpha_{\mathrm{CO}}$ factors intermediate between SB mergers and low- $z$ spirals.

7. The more turbulent galaxy types or regions overall tend to have broader line widths and lower $\alpha_{\mathrm{CO}}$, but the $\alpha_{\mathrm{CO}}$ parameter cannot be simply linked to the bulk ISM turbulent speed, because other properties of ISM turbulence play a substantial role.

Acknowledgements. We are grateful to Desika Narayanan, Mark Sargent, and Anita Zanella for stimulating discussions, to Damien Chapon for help with the implementation of some post-processing modules, and to the anonymous referee for a very careful reading and useful comments. The simulations presented in this work were performed at the Très Grand Centre de Calcul of CEA under GENCI allocations 2013-GEN 2192 and 2014-GEN2192 and on SuperMuc at the LRZ under a PRACE allocation. We acknowledge financial support from the EC through an ERC grant StG-257720 (F.B., F.R.).

\section{References}

Bournaud, F. 2010, Galaxy Wars: Stellar Populations and Star Formation in Interacting Galaxies, 423, 177

Bournaud, F., Elmegreen, B. G., Teyssier, R., Block, D. L., \& Puerari, I. 2010, MNRAS, 409, 1088

Bournaud, F., Chapon, D., Teyssier, R., et al. 2011, ApJ, 730, 4

Bournaud, F., Perret, V., Renaud, F., et al. 2014, ApJ, 780, 57

Ceverino, D., Dekel, A., Mandelker, N., et al. 2012, MNRAS, 420, 3490

Combes, F., García-Burillo, S., Braine, J., et al. 2011, A\&A, 528, A124

Combes, F., Boquien, M., Kramer, C., et al. 2012, A\&A, 539, A67

Daddi, E., Elbaz, D., Walter, F., et al. 2010a, ApJ, 714, L118

Daddi, E., Bournaud, F., Walter, F., et al. 2010b, ApJ, 713, 686

Daddi, E., et al. 2014, A\&A, submitted

Dekel, A., \& Krumholz, M. R. 2013, MNRAS, 432, 455

Di Matteo, P., Combes, F., Melchior, A.-L., \& Semelin, B. 2007, A\&A, 468, 61 
Downes, D., \& Solomon, P. M. 1998, ApJ, 507, 615

Duc, P.-A., Brinks, E., Springel, V., et al. 2000, AJ, 120, 1238

Elbaz, D., Dickinson, M., Hwang, H. S., et al. 2011, A\&A, 533, A119

Ellison, S. L., Nair, P., Patton, D. R., et al. 2011, MNRAS, 416, 2182

Ellison, S. L., Mendel, J. T., Scudder, J. M., Patton, D. R., \& Palmer, M. J. D.

2013, MNRAS, 430, 3128

Elmegreen, D. M., Kaufman, M., Brinks, E., Elmegreen, B. G., \& Sundin, M. 1995, ApJ, 453, 100

Elmegreen, D. M., Elmegreen, B. G., Ravindranath, S., \& Coe, D. A. 2007, ApJ, 658,763

Erb, D. K., Shapley, A. E., Pettini, M., et al. 2006, ApJ, 644, 813

Feldmann, R., Gnedin, N. Y., \& Kravtsov, A. V. 2011, ApJ, 732, 115

Feldmann, R., Gnedin, N. Y., \& Kravtsov, A. V. 2012a, ApJ, 758, 127

Feldmann, R., Gnedin, N. Y., \& Kravtsov, A. V. 2012b, ApJ, 747, 124

Flower, D. R. 2001, MNRAS, 328, 147

Frerking, M. A., Langer, W. D., \& Wilson, R. W. 1982, ApJ, 262, 590

Freundlich, J., Combes, F., Tacconi, L. J., et al. 2013, A\&A, 553, A130

Förster Schreiber, N. M., Shapley, A. E., Genzel, R., et al. 2011, ApJ, 739, 45

Gao, Y., \& Solomon, P. M. 2004, ApJ, 606, 271

García-Burillo, S., Usero, A., Alonso-Herrero, A., et al. 2012, A\&A, 539, A8

Genzel, R., Tacconi, L. J., Gracia-Carpio, J., et al. 2010, MNRAS, 407, 2091

Genzel, R., Newman, S., Jones, T., et al. 2011, ApJ, 733, 101

Genzel, R., Tacconi, L. J., Combes, F., et al. 2012, ApJ, 746, 69

Genzel, R., Tacconi, L. J., Lutz, D., et al. 2014, ApJ, submitted [arXiv: 1409.1171]

Hopkins, P. F., Keres, D., Onorbe, J., et al. 2014, MNRAS, 445, 581

Irwin, J. A. 1994, ApJ, 429, 618

Israel, F. P. 1997, A\&A, 328, 471

Kim, C.-G., Ostriker, E. C., \& Kim, W.-T. 2013, ApJ, 776, 1

Kraljic, K., Renaud, F., Bournaud, F., et al. 2014, ApJ, 784, 112

Krumholz, M. R., \& Gnedin, N. Y. 2011, ApJ, 729, 36

Lacy, J. H., Knacke, R., Geballe, T. R., \& Tokunaga, A. T. 1994, ApJ, 428, L69

Leroy, A. K., Bolatto, A., Gordon, K., et al. 2011, ApJ, 737, 12

Lequeux, J. 2005, The interstellar medium, translation of Le Milieu Interstellaire by James Lequeux (EDP Sciences), 2003, Astronomy and astrophysics library (Berlin: Springer)

Magdis, G. E., Daddi, E., Béthermin, M., et al. 2012, ApJ, 760, 6
Magnelli, B., Lutz, D., Saintonge, A., et al. 2014, A\&A, 561, A86

Meidt, S. E., Schinnerer, E., García-Burillo, S., et al. 2013, ApJ, 779, 45

Narayanan, D., \& Hopkins, P. F. 2013, MNRAS, 433, 1223

Narayanan, D., \& Krumholz, M. R. 2014, MNRAS, 442, 1411

Narayanan, D., Krumholz, M., Ostriker, E. C., \& Hernquist, et al. 2011, MNRAS, 418, 664

Narayanan, D., Krumholz, M. R., Ostriker, E. C., \& Hernquist, L. 2012, MNRAS, 421, 3127

Papadopoulos, P. P., van der Werf, P. P., Xilouris, E. M., et al. 2012, MNRAS, 426, 2601

Papadopoulos, P. P., Zhang, Z.-Y., Xilouris, E. M., et al. 2014, ApJ, 788, 153

Powell, L. C., Bournaud, F., Chapon, D., \& Teyssier, R. 2013, MNRAS, 434 1028

Perret, V., Renaud, F., Epinat, B., et al. 2014, A\&A, 562, A1

Queyrel, J., Contini, T., Pérez-Montero, E., et al. 2009, A\&A, 506, 681

Queyrel, J., Contini, T., Kissler-Patig, M., et al. 2012, A\&A, 539, A93

Renaud, F., Kraljic, K., \& Bournaud, F. 2012, ApJ, 760, L16

Renaud, F., Bournaud, F., Emsellem, E., et al. 2013, MNRAS, 436, 1836

Renaud, F., Bournaud, F., Kraljic, K., \& Duc, P.-A. 2014a, MNRAS, 442, L33

Renaud, F., Bournaud, F., \& Duc, P.-A. 2014b, MNRAS, submitted Rodighiero, G., Daddi, E., Baronchelli, I., et al. 2011, ApJ, 739, L40

Salmi, F., Daddi, E., Elbaz, D., et al. 2012, ApJ, 754, L14

Saintonge, A., Tacconi, L. J., Fabello, S., et al. 2012, ApJ, 758, 73

Saintonge, A., Lutz, D., Genzel, R., et al. 2013, ApJ, 778, 2

Sargent, M. T., Béthermin, M., Daddi, E., \& Elbaz, D. 2012, ApJ, 747, L31

Sargent, M. T., Daddi, E., Béthermin, M., et al. 2014, ApJ, 793, 19

Schinnerer, E., Meidt, S. E., Pety, J., et al. 2013, ApJ, 779, 42

Solomon, P. M., \& Vanden Bout, P. A. 2005, ARA\&A, 43, 677

Tacconi, L. J., Genzel, R., Neri, R., et al. 2010, Nature, 463, 781

Tacconi, L. J., Neri, R., Genzel, R., et al. 2013, ApJ, 768, 74

Teyssier, R. 2002, A\&A, 385, 337

Teyssier, R., Chapon, D., \& Bournaud, F. 2010, ApJ, 720, L149

Walter, F., Decarli, R., Sargent, M., et al. 2014, ApJ, 782, 79

Wei, L. H., Keto, E., \& Ho, L. C. 2012, ApJ, 750, 136

Weiß, A., Walter, F., \& Scoville, N. Z. 2005, A\&A, 438, 533

Wuyts, E., Kurk, J., Förster Schreiber, N. M., et al. 2014, ApJ, 789, L40 